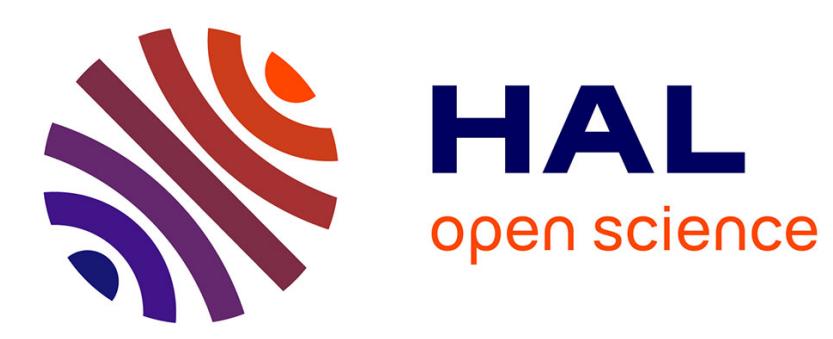

\title{
Real and complex hedgehogs, their symplectic area, curvature and evolutes
}

\author{
Yves Martinez-Maure
}

\section{To cite this version:}

Yves Martinez-Maure. Real and complex hedgehogs, their symplectic area, curvature and evolutes. 2021. hal-02948386v2

\section{HAL Id: hal-02948386 \\ https://hal.science/hal-02948386v2}

Preprint submitted on 8 Jul 2021

HAL is a multi-disciplinary open access archive for the deposit and dissemination of scientific research documents, whether they are published or not. The documents may come from teaching and research institutions in France or abroad, or from public or private research centers.
L'archive ouverte pluridisciplinaire HAL, est destinée au dépôt et à la diffusion de documents scientifiques de niveau recherche, publiés ou non, émanant des établissements d'enseignement et de recherche français ou étrangers, des laboratoires publics ou privés. 


\title{
Real and complex hedgehogs, their symplectic area, curvature and evolutes
}

\author{
Yves Martinez-Maure \\ yves.martinez-maure@imj-prg.fr
}

\begin{abstract}
Classical (real) hedgehogs can be regarded as the geometrical realizations of formal differences of convex bodies in $\mathbb{R}^{n+1}$. Like convex bodies, hedgehogs can be identified with their support functions. Adopting a projective viewpoint, we prove that any holomorphic function $h: \mathbb{C}^{n} \rightarrow \mathbb{C}$ can be regarded as the 'support function' of a complex hedgehog $\mathcal{H}_{h}$ in $\mathbb{C}^{n+1}$. In the same vein, we introduce the notion of evolute of such a hedgehog $\mathcal{H}_{h}$ in $\mathbb{C}^{2}$, and a natural (but apparently hitherto unknown) notion of complex curvature, which allows us to interpret this evolute as the locus of the centers of complex curvature. It is of course permissible to think that the development of a 'Brunn-Minkowski theory for complex hedgehogs' (replacing Euclidean volumes by symplectic ones) might be a promising way of research. We give first two results in this direction. We next return to real hedgehogs in $\mathbb{R}^{2 n}$ endowed with a linear complex structure. We introduce and study the notion of evolute of a hedgehog. We particularly focus our attention on $\mathbb{R}^{4}$ endowed with a linear Kähler structure determined by the datum of a pure unit quaternion. In parallel, we study the symplectic area of the images of the oriented Hopf circles under hedgehog parametrizations and introduce a quaternionic curvature function for such an image. Finally, we consider briefly the convolution of hedgehogs, and the particular case of hedgehogs in $\mathbb{R}^{4 n}$ regarded as a hyperkähler vector space.
\end{abstract}

\section{Contents:}

1. Introduction

2. Background on classical real hedgehogs

3. Complex hedgehogs in $\mathbb{C}^{n+1}$ or $P^{n+1}(\mathbb{C})$

3.1. Real and complex hedgehogs as dual hypersurfaces of graphs

2010 MSC:

Key words and phrases: Real and complex hedgehogs, Brunn-Minkowski theory, mixed symplectic areas, evolutes, rational curves, complex or quaternionic curvature function, linear complex structure, Kähler vector spaces 
3.2. Complex hedgehogs as fronts in $\mathbb{C}^{n+1}$ of Legendrian immersions in $\mathbb{C}^{2 n+1}$

3.3. Rational hedgehogs of the complex projective plane $P^{2}(\mathbb{C})$

3.4. Evolute of a plane complex hedgehog as locus of its centers of curvature

3.5 Real and imaginary parts of $\mathcal{H}_{h} \subset \mathbb{C}^{2}$ regarded as hedgehogs of $\mathbb{R}^{3}$

4. Towards a Brunn-Minkowski theory for complex hedgehogs

4.1. Mixed symplectic area

4.2. A sharp estimation of the area using the energy

5. Real hedgehogs in $\mathbb{C}^{n} \cong \mathbb{R}^{2 n}$ and their evolutes

5.1. Evolutes of hedgehogs hypersurfaces in $\mathbb{R}^{4}$

5.2. Symplectic and mixed symplectic area

5.3. Quaternionic curvature function

5.4. Convolution of hedgehogs

5.5. Evolutes of hedgehogs hypersurfaces in $\mathbb{H}^{n} \cong \mathbb{R}^{4 n}$

\section{Introduction}

Classical (real) hedgehogs can be regarded as the geometrical realizations of formal differences of convex bodies in the Euclidean vector space $\mathbb{R}^{n+1}$. The idea of considering the Minkowski differences of convex bodies may be traced back to some papers by A.D. Alexandrov [1] and H. Geppert [4] in the 1930's. Many notions extend to hedgehogs and quite a number of classical results find their counterparts. Of course, a few adaptations are necessary. In particular, volumes have to be replaced by their algebraic versions. Hedgehogs have proved useful for studying convex bodies (one of the main successes of the theory is the construction of counterexamples to an old conjectured characterization of the 2-sphere [11, 15]), and for geometrizing analytical problems by considering functions as support functions. Section 2 will provide the reader with the necessary background on hedgehogs in order to facilitate an understanding of the following sections.

\section{Complex hedgehogs}

Like convex bodies of $\mathbb{R}^{n+1}$, hedgehogs of $\mathbb{R}^{n+1}$ are completely determined by (and can be identified with) their support functions, which are differences of two support functions of convex bodies of $\mathbb{R}^{n+1}$ restricted to the unit sphere $\mathbb{S}^{n}$. In section 3, we adopt a projective viewpoint in order to introduce the notion of a 'complex hedgehog' in the complex Euclidean space $\mathbb{C}^{n+1}$. We prove that:

Any holomorphic function $h: \mathbb{C}^{n} \rightarrow \mathbb{C}$ can be regarded as the 'complex support function' of a 'complex hedgehog' $\mathcal{H}_{h}$, which is defined by a holomorphic parametrization $x_{h}: \mathbb{C}^{n} \rightarrow \mathbb{C}^{n+1}$ in the complex Euclidean space $\mathbb{C}^{n+1}$.

Of course, these complex hedgehogs can be interpreted in the metric contact geometry setting where they appear as fronts of Legendrian immersions in $\mathbb{C}^{2 n+1}$ (see Subsection 3.2). 
In passing, we introduce the notion of a rational hedgehog in the complex projective plane $P^{2}(\mathbb{C})$ equipped with the usual Fubini-Study Kähler form $\omega$ (for an introduction to the Fubini-Study structure, see e.g. [2]). Such a hedgehog $\mathcal{H}_{h}$ is modeled on $P^{1}(\mathbb{C}):=\mathbb{C} \cup\{\infty\}$ via a holomorphic map $h: \mathbb{C} \rightarrow \mathbb{C}$ that is such that Area $\left[x_{h}(\mathbb{C})\right]<+\infty$.

Complex evolutes and complex curvature

In classical differential geometry of curves, the evolute of a plane curve is the locus of all its centers of curvature or, equivalently, the envelope of its normal lines. Interpreting evolutes of hedgehog curves from a projective point of view, we prove in Subsection 3.4 that:

There exists a natural extension of the notion of evolute curves to complex hedgehog curves, and a very natural (but apparently hitherto unknown) notion of complex curvature, which allows us to interpret any evolute of a complex hedgehog curve $\mathcal{H}_{h}$ as the locus of its centers of complex curvature.

Given any complex hedgehog $\mathcal{H}_{h}$ in $\mathbb{R}^{4}$, we introduce its real and imaginary parts as hedgehogs of $\mathbb{R}^{3}$, which can be regarded globally as the images of $\mathcal{H}_{h}$ under the orthogonal projections onto two particular hyperplanes of $\mathbb{R}^{4}$, and that are determined by $\operatorname{Re}[h]$ and $\operatorname{Im}[h]$.

Towards a Brunn-Minkowski theory for complex hedgehogs

The notion of a hedgehog curve or surface was born in the thirties from the study of the Brunn-Minkowski theory by A.D. Aleksandrov, H. Geppert and some others. In the present paper, we try to motivate the development of a 'theory of mixed volumes for complex hedgehogs' (replacing Euclidean volumes by symplectic ones).

In Section 4, we mention first two results in this direction. First, identifying complex hedgehogs with their support functions, we notice that the complex linear space of holomorphic functions defined up to a similitude on the unit disc $\mathbb{D} \subset \mathbb{C}$ can be endowed with a scalar product which can be interpreted as a mixed symplectic area.

Second, we give the following sharp estimation of the (symplectic) area of $x_{h}(\mathbb{D})$ using the energy, say $E\left(x_{h}\right)$, of the loop $x_{h}: \mathbb{S}^{1}=\mathbb{R} / 2 \pi \mathbb{Z} \rightarrow \mathbb{C}^{2}$, $\theta \mapsto x_{h}\left(e^{i \theta}\right)$, in the case where $h: \mathbb{D} \rightarrow \mathbb{C}$ is the sum of a power series $\sum h_{n} z^{n}$ with radius of convergence $R>1$ :

$$
\text { Area }\left[x_{h}(\mathbb{D})\right] \leq \frac{3}{4} E\left(x_{h}\right) .
$$

Note that this estimate is better than that well-known for an arbitrary smooth loop $\gamma: \mathbb{S}^{1} \rightarrow V$ in a symplectic vector space $(V, \omega)$ (namely, $|A(\gamma)| \leq E(\gamma)$, see for instance [14, pp. 87-88]).

Real evolutes in even dimensions 
In Section 5 , we return to real hedgehogs but in $\mathbb{R}^{2 n}$ endowed with a linear complex structure $J$. First of all, we introduce the notion of evolute of any hedgehog with a smooth support function in $\left(\mathbb{R}^{2 n}, J\right)$.

We particularly focus our attention on the case $n=2$. We identify $\mathbb{R}^{4}$ with the quaternion algebra $\mathbb{H}$ (and thus the unit sphere $\mathbb{S}^{3}$ with the set $\mathbb{S}_{\mathbb{H}}^{1}$ of unit quaternions), and, we associate to any pure unit quaternion $v$ the linear complex structure $J_{v}: \mathbb{R}^{4} \rightarrow \mathbb{R}^{4}, x \longmapsto v x$. In other words, for any $v \in \mathbb{S}^{2} \cong \mathbb{S}_{\mathbb{H}}^{1} \cap \operatorname{Im}(\mathbb{H})$, we choose to work in the Kähler vector space $\left(\mathbb{R}^{4}, J_{v}, \omega_{v}\right)$, where $\omega_{v}$ denotes the associated Kähler form (i.e. the alternating 2-form $\omega_{v}(X, Y)=\left\langle J_{v} X, Y\right\rangle$, where $\langle.,$.$\left.\rangle is the standard Euclidean metric on \mathbb{R}^{4}\right)$. To any $v \in \mathbb{S}^{2}$, it thus corresponds a Hopf fibration and a Hopf flow leaving the Hopf fibration invariant, namely the Hopf flow $\left\{\left(\phi_{v}\right)_{\theta}\right\}_{\theta \in \mathbb{S}^{1}}$ given by $\left(\phi_{v}\right)_{\theta}(u):=(\cos \theta) u+(\sin \theta) v u,\left(u \in \mathbb{S}^{3}\right)$.

We give a detailed study of evolutes of hedgehog hypersurfaces in these Kähler vector spaces $\left(\mathbb{R}^{4}, J_{v}, \omega_{v}\right)$.

Mixed symplectic area and quaternionic curvature function

In parallel, we study the symplectic area of images of the oriented Hopf circles under the hedgehog parametrizations $x_{h}: \mathbb{S}^{3} \rightarrow\left(\mathbb{R}^{4}, J_{v}, \omega_{v}\right)$. In this setting, we introduce the notion of mixed symplectic area and prove what follows among other results.

Theorem. Let $h \in C^{\infty}\left(\mathbb{S}^{3} ; \mathbb{R}\right)$, and let $v$ be a pure unit quaternion.

(i) The evolute of $\mathcal{H}_{h}$ in $\left(\mathbb{R}^{4}, J_{v}, \omega_{v}\right)$ is the hedgehog with support function

$$
\partial_{v} h: \mathbb{S}^{3} \rightarrow \mathbb{R}, u \longmapsto\left\langle\nabla h\left(-J_{v}(u)\right), u\right\rangle,
$$

where $\langle.,$.$\rangle is the standard Euclidean metric on \mathbb{R}^{4}$, and $\nabla h$ the gradient of $h$. Thus, $\partial_{v} h$ is such that: $\forall u \in \mathbb{S}^{3}$,

$$
\left(\partial_{v} h\right)\left(J_{v}(u)\right)=\left\langle\nabla h(u), J_{v}(u)\right\rangle=(d h)_{u}\left(J_{v}(u)\right) ;
$$

(ii) For all $u \in \mathbb{S}^{3}$,

$$
x_{\partial_{v} h}(u)=x_{h}(u)-R_{h}(u, v) u,
$$

where $R_{h}(u, v):=-v T_{u} x_{h}\left(J_{v}(u)\right) \bar{u}$; here $\bar{u}$ of course refers to the quaternion conjugate of $u$;

(iii) The map $R_{h}(., v): u_{\theta}:=(\cos \theta) u+(\sin \theta) v u \longmapsto R_{h}\left(u_{\theta}, v\right)$ can be interpreted as a quaternionic curvature function of $x_{h}\left(\mathbb{S}_{u, v}^{1}\right)$, where $\mathbb{S}_{u, v}^{1}$ is the unit circle of $\mathbb{C}(u, v):=\mathbb{R} u+\mathbb{R} J_{v}(u)$ oriented by $\left(u, J_{v}(u)\right)$, in the sense that $R_{h}(., v)$ is the unique $C^{\infty}$-smooth quaternionic function $R(., v): \mathbb{S}_{u, v}^{1} \rightarrow \mathbb{H}$ that is of the form $R\left(u_{\theta}, v\right)=-v T_{u_{\theta}}(v)$, where $T_{u_{\theta}}(v)$ is a pure quaternion, and such that:

$$
\forall g \in C^{\infty}\left(\mathbb{S}^{3} ; \mathbb{R}\right), \quad s_{u, v}(g, h):=\frac{1}{2} \int_{0}^{2 \pi}\left\langle x_{g}\left(u_{\theta}\right), R\left(u_{\theta}, v\right) u_{\theta}\right\rangle d \theta,
$$

where $s_{u, v}(g, h)$ denotes the mixed symplectic area of $x_{g}\left(\mathbb{S}_{u, v}^{1}\right)$ and $x_{h}\left(\mathbb{S}_{u, v}^{1}\right)$. 
In other words, what is shown by (iii) is that the quaternionic curvature function $R(., v)$ plays, relatively to the mixed symplectic area $s_{u, v}$, the same role as the (ordinary) curvature function of plane hedgehogs does relatively to the (ordinary) mixed area. Here, we have to recall that the mixed area of two plane hedgehogs with support functions $(g, h) \in C^{\infty}\left(\mathbb{S}^{1} ; \mathbb{R}\right)^{2}$ is given by

$$
a(g, h):=\frac{1}{2} \int_{0}^{2 \pi}\left\langle x_{g}\left(u_{\theta}\right), R_{h}(u) u_{\theta}\right\rangle d \theta=\frac{1}{2} \int_{0}^{2 \pi} g\left(u_{\theta}\right) R_{h}\left(u_{\theta}\right) d \theta,
$$

where $u_{\theta}=e^{i \theta} \in \mathbb{C} \cong \mathbb{R}^{2}$, and where $x_{g}: \mathbb{S}^{1} \rightarrow \mathbb{C}, \theta \mapsto g(\theta) u_{\theta}+g^{\prime}(\theta) i u_{\theta}$ is the natural parametrization of $\mathcal{H}_{g}$, and $R_{h}:=h+h^{\prime \prime}$ the so-called 'curvature function' of $\mathcal{H}_{h}$ (see $[9$, p. 447]).

Relationship with the area of order 2

We also show that the algebraic area of order 2 of a hedgehog $\mathcal{H}_{h}$ of $\mathbb{R}^{4}$ can be interpreted in terms of the symplectic areas of $\mathcal{H}_{h}$ in the Kähler vector spaces $\left(\mathbb{R}^{4}, J_{v}, \omega_{v}\right)$. Here, we have to recall that the algebraic area of order 2 of $\mathcal{H}_{h}$ is defined to be $V(h, h, 1,1)$, where $V$ is the extension of the mixed volume (of convex bodies of $\mathbb{R}^{4}$ ) to hedgehogs of $\mathbb{R}^{4}$.

Convolution and extension to $\mathbb{R}^{4 n} \cong \mathbb{H}^{n}$

Finally, we consider briefly the convolution of hedgehogs in $\mathbb{R}^{n}$, and evolutes of hedgehog hypersurfaces in $\mathbb{R}^{4 n}$, which we identify with the hyperkähler vector space $\left(\mathbb{H}^{n},\langle.,\rangle, I, J, K.\right)$, where $\langle.,$.$\rangle is the standard Euclidean metric on \mathbb{R}^{4 n}$, $(n \geq 1)$, and, the triple of complex structures $(I, J, K)$ on $\mathbb{H}^{n}$ is given by left multiplication by $i, j, k$ respectively.

\section{Background on classical real hedgehogs}

In this section, we recall for the convenience of the reader the background on real hedgehogs. The set $\mathcal{K}^{n+1}$ of all convex bodies of $(n+1)$-Euclidean vector space $\mathbb{R}^{n+1}$ is usually equipped with Minkowski addition and multiplication by nonnegative real numbers, which are respectively defined by:

(i) $\forall(K, L) \in\left(\mathcal{K}^{n+1}\right)^{2}, K+L=\{x+y \mid x \in K, y \in L\}$;

(ii) $\forall \lambda \in \mathbb{R}_{+}, \forall K \in \mathcal{K}^{n+1}, \lambda . K=\{\lambda x \mid x \in K\}$.

It does not constitute a vector space since there is no subtraction in $\mathcal{K}^{n+1}$ : not for every pair $(K, L) \in\left(\mathcal{K}^{n+1}\right)^{2}$ does there exist an $X \in \mathcal{K}^{n+1}$ such that $L+X=K$. Now, in the same way as we construct the group $\mathbb{Z}$, of integers from the monoid $\mathbb{N}$ of nonnegative integers, we can construct the vector space $\mathcal{H}^{n+1}$ of formal differences of convex bodies from $\mathcal{K}^{n+1}$. We can then regard $\mathcal{K}^{n+1}$ as a cone of $\mathcal{H}^{n+1}$ that spans the entire space. Hedgehog theory simply consists in: 
1. considering each formal difference of convex bodies of $\mathbb{R}^{n+1}$ as a geometrical object in $\mathbb{R}^{n+1}$, called a hedgehog (see below);

2. extending the mixed volume $V:\left(\mathcal{K}^{n+1}\right)^{n+1} \rightarrow \mathbb{R}$ to a symmetric $(n+1)$ linear form on $\mathcal{H}^{n+1}$;

3. extending certain parts of the Brunn-Minkowski theory to $\mathcal{H}^{n+1}$.

For $n \leq 2$, it goes back to a paper by H. Geppert [4] who introduced hedgehogs under the German names stützbare Bereiche $(n=1)$ and stützbare Flächen $(n=2)$.

$\mathbf{C}^{2}$ case. Here we follow more or less [8]. As is well-known, every convex body $K \subset \mathbb{R}^{n+1}$ is determined by its support function $h_{K}: \mathbb{S}^{n} \longrightarrow \mathbb{R}$, where $h_{K}(u)$ is defined by $h_{K}(u)=\sup \{\langle x, u\rangle \mid x \in K\},\left(u \in \mathbb{S}^{n}\right)$, that is, as the signed distance from the origin to the support hyperplane with normal vector $u$. In particular, every closed convex hypersurface of class $C_{+}^{2}$ (i.e., $C^{2}$-hypersurface with positive Gaussian curvature) is determined by its support function $h$ (which must be of class $C^{2}$ on $\mathbb{S}^{n}\left[16\right.$, p. 111]) as the envelope $\mathcal{H}_{h}$ of the family of hyperplanes with equation $\langle x, u\rangle=h(u)$. This envelope $\mathcal{H}_{h}$ is described analytically by the following system of equations

$$
\left\{\begin{array}{c}
\langle x, u\rangle=h(u) \\
\langle x, .\rangle=d h_{u}(.)
\end{array} .\right.
$$

The second equation is obtained from the first by performing a partial differentiation with respect to $u$. From the first equation, the orthogonal projection of $x$ onto the line spanned by $u$ is $h(u) u$, and from the second one, the orthogonal projection of $x$ onto $u^{\perp}$ is the gradient of $h$ at $u$ (see Figure 1). Therefore, for each $u \in \mathbb{S}^{n}, x_{h}(u)=h(u) u+(\nabla h)(u)$ is the unique solution of this system. 


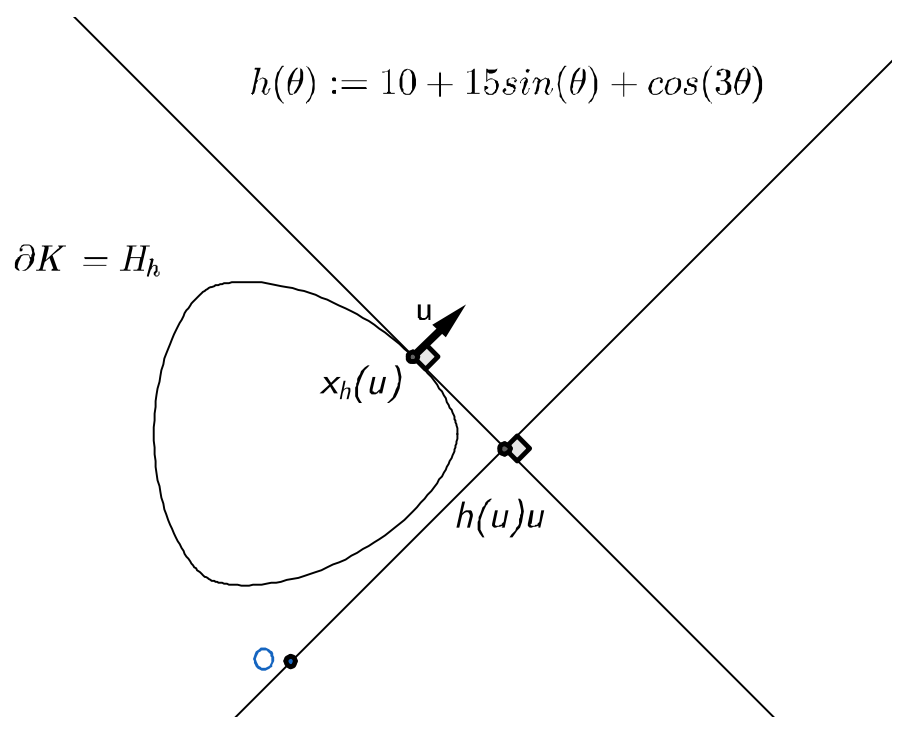

Figure 1. Envelope parametrized by its Gauss map

Now, for any $C^{2}$-function $h$ on $\mathbb{S}^{n}$, the envelope $\mathcal{H}_{h}$ is in fact well-defined (even if $h$ is not the support function of a convex hypersurface). Its natural parametrization $x_{h}: \mathbb{S}^{n} \rightarrow \mathcal{H}_{h}, u \mapsto h(u) u+(\nabla h)(u)$ can be interpreted as the inverse of its Gauss map, in the sense that: at each regular point $x_{h}(u)$ of $\mathcal{H}_{h}, u$ is a normal vector to $\mathcal{H}_{h}$. We say that $\mathcal{H}_{h}$ is the hedgehog with support function $h$ (see Figure 2). Note that $x_{h}$ depends linearly on $h$.

Since the parametrization $x_{h}$ can be regarded as the inverse of the Gauss map, the Gaussian curvature $K_{h}$ of $\mathcal{H}_{h}$ at $x_{h}(u)$ is given by $K_{h}(u)=1 / \operatorname{det}\left[T_{u} x_{h}\right]$, where $T_{u} x_{h}$ is the tangent map of $x_{h}$ at $u$. Therefore, singularities are the very points at which the Gaussian curvature is infinite. For every $u \in \mathbb{S}^{n}$, the tangent map of $x_{h}$ at the point $u$ is $T_{u} x_{h}=h(u) I d_{T_{u} \mathbb{S}^{n}}+H_{h}(u)$, where $H_{h}(u)$ is the symmetric endomorphism associated with the Hessian $\left(\nabla^{2} h\right)_{u}$ of $h$ at $u$. In particular, the so-called 'curvature function' $R_{h}(u):=\operatorname{det}\left[T_{u} x_{h}\right]$ is given by $R_{h}(u)=\operatorname{det}\left[h(u) I d_{T_{u} \mathbb{S}^{n}}+H_{h}(u)\right]$ for all $u \in \mathbb{S}^{n}$. 


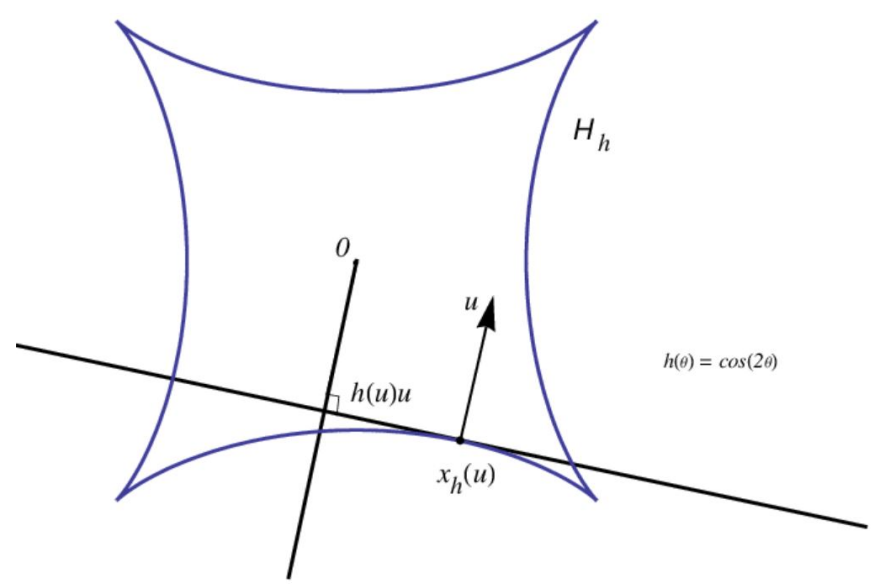

Figure 2. Plane hedgehog with $C^{2}$-support function

In computations, it is often more convenient to replace $h$ by its positively 1 -homogeneous extension to $\mathbb{R}^{n+1} \backslash\{0\}$, which is given by

$$
\varphi(x):=\|x\| h\left(\frac{x}{\|x\|}\right),
$$

for $x \in \mathbb{R}^{n+1} \backslash\{0\}$, where $\|\cdot\|$ is the Euclidean norm on $\mathbb{R}^{n+1}$. A straightforward computation gives:

(i) $x_{h}$ is the restriction of the Euclidean gradient of $\varphi$ to the unit sphere $\mathbb{S}^{n}$;

(ii) For all $u \in \mathbb{S}^{n}$, the tangent map $T_{u} x_{h}$ identifies with the symmetric endomorphism associated with the Hessian of $\varphi$ at $u$.

Hedgehogs with a $C^{2}$-support function can be regarded as Minkowski differences of convex hypersurfaces of class $C_{+}^{2}$. Indeed, given any $h \in C^{2}\left(\mathbb{S}^{n} ; \mathbb{R}\right)$, for all large enough real constants $r$, the functions $h+r$ and $r$ are support functions of convex hypersurfaces of class $C_{+}^{2}$ such that $h=(h+r)-r$.

General case. In [12], the author extended the notion of a hedgehog by regarding hedgehogs as Minkowski differences of arbitrary convex bodies. The trick is to define hedgehogs inductively as collections of lower-dimensional 'support hedgehogs'. More precisely, the definition of general hedgehogs is based on the three following remarks.

(i) In $\mathbb{R}$, every convex body $K$ is determined by its support function $h_{K}$ as the segment $\left[-h_{K}(-1), h_{K}(1)\right]$, where $-h_{K}(-1) \leq h_{K}(1)$, so that the difference $K-L$ of two convex bodies $K, L$ can be defined as an oriented segment of $\mathbb{R}$ : $K-L:=\left[-\left(h_{K}-h_{L}\right)(-1),\left(h_{K}-h_{L}\right)(1)\right]$.

(ii) If $K$ and $L$ are two convex bodies of $\mathbb{R}^{n+1}$ then for all $u \in \mathbb{S}^{n}$, their support sets with unit normal $u$, say $K_{u}$ and $L_{u}$, can be identified with convex bodies $K_{u}$ and $L_{u}$ of the $n$-dimensional Euclidean vector space $u^{\perp} \simeq \mathbb{R}^{n}$. 
(iii) Addition of two convex bodies $K, L \subset \mathbb{R}^{n+1}$ corresponds to that of their support sets with same unit normal vector: $(K+L)_{u}=K_{u}+L_{u}$ for all $u \in \mathbb{S}^{n}$; therefore, the difference $K-L$ of two convex bodies $K, L \subset \mathbb{R}^{n+1}$ must be defined in such a way that $(K-L)_{u}=K_{u}-L_{u}$ for all $u \in \mathbb{S}^{n}$.

A natural way of defining geometrically general hedgehogs as differences of arbitrary convex bodies is therefore to proceed by induction on the dimension by extending the notion of support set with normal vector $u$ to a notion of support hedgehog with normal vector $u$. Let us give an example in $\mathbb{R}^{2}$. Let $K$ and $L$ be the convex bodies of $\mathbb{R}^{2}$ with support function $h_{K}(x)=\left|\left\langle x, e_{1}\right\rangle\right|+\left|\left\langle x, e_{2}\right\rangle\right|$ and $h_{L}(x)=\left|\left\langle x, e_{3}\right\rangle\right|+\left|\left\langle x, e_{4}\right\rangle\right|$, where $\langle.,$.$\rangle is the standard inner product on \mathbb{R}^{2}$, $\left(e_{1}, e_{2}\right)$ the canonical basis of $\mathbb{R}^{2}$ and $e_{3}, e_{4} \in \mathbb{R}^{2}$ the unit vectors given by $e_{3}=\frac{1}{\sqrt{2}}\left(e_{1}+e_{2}\right)$ and $e_{4}=\frac{1}{\sqrt{2}}\left(e_{1}-e_{2}\right)$. These convex bodies are two squares whose formal difference $K-L$ can be realized geometrically as the hedgehog with support function $h=h_{K}-h_{L}$, which is a regular octagram constructed by connecting every third consecutive vertex of a regular octogon (i.e., a regular star polygon with Schläfli symbol $\{8 / 3\})$ : see Figure 3 .

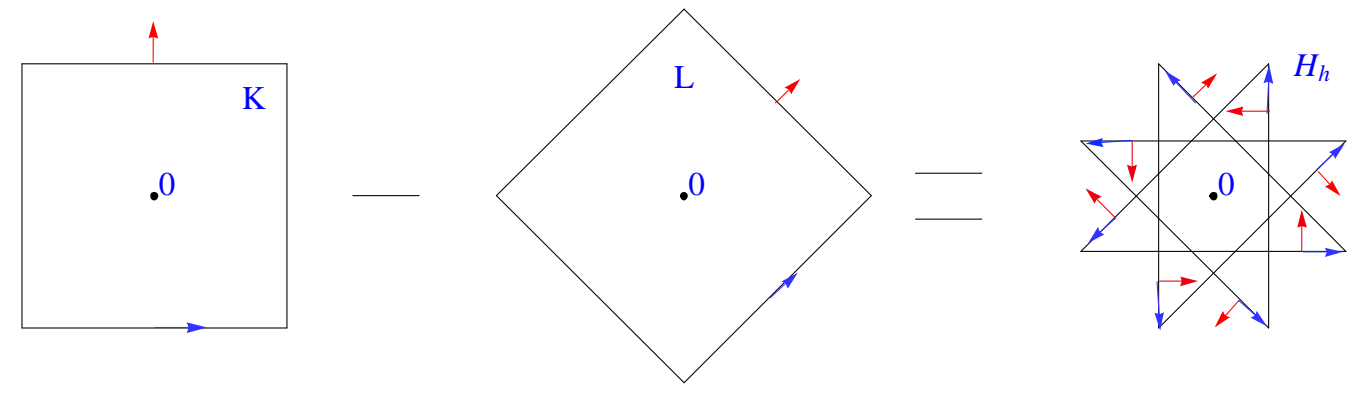

Figure 3. Octagram obtained as the difference of two squares

Polytopal hedgehogs and hedgehogs with an analytical support function can also be introduced in index terms via Euler Calculus [13].

\section{Complex hedgehogs in $\mathbb{C}^{n+1}$ or $P^{n+1}(\mathbb{C})$}

\subsection{Real and complex hedgehogs as dual hypersurfaces of graphs}

In order to introduce complex hedgehogs, it is convenient to recall that real hedgehogs with a smooth support function can be regarded as dual hypersurfaces of smooth graphs. In what follows, any hedgehog $\mathcal{H}_{h} \subset \mathbb{R}^{n+1}$ with support function $h \in C^{\infty}\left(\mathbb{S}^{n} ; \mathbb{R}\right)$ will be regarded as a hypersurface in the real projective space $P^{n+1}(\mathbb{R})$ by adding 'a hyperplane at the infinity' $H_{\infty}$ to $\mathbb{R}^{n+1}$ : 
$P^{n+1}(\mathbb{R})=\mathbb{R}^{n+1} \cup H_{\infty}$. More precisely, we will identify $\mathbb{R}^{n+1}$ with the affine hyperplane of $P^{n+1}(\mathbb{R})=\mathbb{R}^{n+2}-\{0\} / \mathbb{R}^{*}$ with equation $X_{n+2}=-1$, where $\left[X_{1}, \ldots, X_{n+2}\right]$ denote the homogeneous coordinates of the equivalent class of $\left(X_{1}, \ldots, X_{n+2}\right) \in \mathbb{R}^{n+2} \backslash\{0\}$ in $P^{n+1}(\mathbb{R})$. Then, the hedgehog hypersurface $x_{h}: \mathbb{S}^{n} \rightarrow \mathcal{H}_{h} \subset \mathbb{R}^{n+1} \subset P^{n+1}(\mathbb{R})$ can be regarded as the dual hypersurface of

$$
\begin{aligned}
& \gamma_{h}: \mathbb{S}^{n} \subset \mathbb{R}^{n+1} \quad \rightarrow P^{n+1}(\mathbb{R}) \\
& u=\left(u_{1}, \ldots, u_{n+1}\right) \mapsto\left[u_{1}, \ldots, u_{n+1}, h(u)\right] .
\end{aligned}
$$

Indeed, the support hyperplane with equation $\langle x, u\rangle=h(u)$ then corresponds to the point $\gamma_{h}(u)$ by projective duality.

It is extremely natural to follow this idea to extend the notion of hedgehog to the complex setting. We regard the complex Euclidean space $\mathbb{C}^{n+1}$ as the affine hyperplane of $P^{n+1}(\mathbb{C})=\mathbb{C}^{n+2}-\{0\} / \mathbb{C}^{*}$ with equation $X_{n+2}=-1$, and we define, for any holomorphic function $h: \mathbb{C}^{n} \rightarrow \mathbb{C}$, the hedgehog with support function $h$ as the hypersurface of $\mathbb{C}^{n+1}$ that is the dual hypersurface of

$$
\begin{aligned}
\gamma_{h}: \mathbb{C}^{n} & \rightarrow P^{n+1}(\mathbb{C}) \\
z=\left(z_{1}, \ldots, z_{n}\right) & \mapsto\left[1, z_{1}, \ldots, z_{n}, h(z)\right],
\end{aligned}
$$

that is, as the envelope of the family of hyperplanes $\left(H_{h}(z)\right)_{z \in \mathbb{C}^{n}}$ with equation

$$
X_{1}+\sum_{k=1}^{n} z_{k} X_{k+1}=h(z) \text {. }
$$

In other words:

Definition 1 Let $h: \mathbb{C}^{n} \rightarrow \mathbb{C}$ be a holomorphic function. The hypersurface $\mathcal{H}_{h}$ of the complex Euclidean space $\mathbb{C}^{n+1}$ that is parametrized by

$$
\begin{aligned}
x_{h}: \mathbb{C}^{n} & \rightarrow \mathbb{C}^{n+1} \\
z=\left(z_{1}, \ldots, z_{n}\right) & \mapsto\left(h(z)-\sum_{k=1}^{n} z_{k} \frac{\partial h}{\partial z_{k}}(z), \frac{\partial h}{\partial z_{1}}(z), \ldots, \frac{\partial h}{\partial z_{n}}(z)\right)
\end{aligned}
$$

is called the hedgehog with support function $h$.

Indeed, from (1) and the contact condition $d w_{0}+\sum_{j=1}^{n} z_{j} d w_{j}=0$, where $\left(w_{0}, w_{1}, \ldots, w_{n}, z_{1}, \ldots, z_{n}\right) \in \mathbb{C}^{n+1} \times \mathbb{C}^{n}=\mathbb{C}^{2 n+1}$, we deduce that for all $z \in \mathbb{C}^{n}$, the point $x_{h}(z)=\left(x_{1}(z), \ldots, x_{n}(z)\right)$ is the unique solution of the system

$$
\left\{\begin{array}{l}
x_{1}+\sum_{k=1}^{n} z_{k} x_{k+1}=h(z) \\
\forall k \in\{1, \ldots, n\}, x_{k+1}=\frac{\partial h}{\partial z_{k}}(z)
\end{array}\right.
$$

where (2) is obtained from (1) by performing partial differentiations with respect to the complex variables $z_{k},(1 \leq k \leq n)$. Thus, it appears that $\mathcal{H}_{h}$ is actually parametrized by 
$x_{h}: \mathbb{C}^{n} \rightarrow \mathbb{C}^{n+1}, z=\left(z_{1}, \ldots, z_{n}\right) \mapsto\left(h(z)-\sum_{k=1}^{n} z_{k} \frac{\partial h}{\partial z_{k}}(z), \frac{\partial h}{\partial z_{1}}(z), \ldots, \frac{\partial h}{\partial z_{n}}(z)\right)$

Example. The hedgehog of $\mathbb{C}^{2}$ of which the support function $h: \mathbb{C} \rightarrow \mathbb{C}$ is given by $h(z)=-z^{3}$ is the affine algebraic curve $\mathcal{H}_{h}$ of $\mathbb{C}^{2}$ with equation $27 x^{2}+4 y^{3}=0$. It is parametrized by:

$$
\left\{\begin{array}{l}
x=2 z^{3} \\
y=-3 z^{2} .
\end{array}\right.
$$

As any complex hedgehog curve $x_{h}: \mathbb{C} \rightarrow \mathbb{C}^{2}$, it is such that:

$$
\forall z \in \mathbb{C}, \quad x_{h}^{\prime}(z)=-h^{\prime \prime}(z)(z,-1) \in \mathbb{C}(z,-1) .
$$

Naturally, we could have introduced complex hedgehogs of $\mathbb{C}^{n+1}$ in the complex contact geometry setting, where they appear as fronts of Legendrian immersions in $\mathbb{C}^{2 n+1}$ (see the next subsection).

Remark. Of course, many other parametrizations would have been possible in order to introduce the notion of a complex hedgehog. New parametrizations can simply be obtained by performing chart changes. For instance, for any holomorphic function $g: \mathbb{C} \rightarrow \mathbb{C}$, the complex curve

$$
y_{g}: \mathbb{C} \rightarrow \mathbb{C}, x_{h}: z \longmapsto\left(g^{\prime}(z), g(z)-z g^{\prime}(z)\right)
$$

is a hedgehog, namely the hedgehog with support function $f(z)=z g(1 / z)$ :

$$
\forall z \in \mathbb{C}^{*}, y_{g}(z)=x_{f}\left(\frac{1}{z}\right) .
$$

Therefore, this particular parametrization change only corresponds to the chart change $z \mapsto 1 / z$ on the Riemann sphere $P^{1}(\mathbb{C})=\mathbb{C} \cup\{\infty\}$.

\subsection{Complex hedgehogs as fronts in $\mathbb{C}^{n+1}$ of Legendrian immersions in $\mathbb{C}^{2 n+1}$}

Consider the complex Euclidean space $\mathbb{C}^{2 n+1}$ endowed with the holomorphic contact form

$$
\omega:=d w_{0}+\sum_{j=1}^{n} z_{j} d w_{j},
$$

where $\left(w_{0}, w_{1}, \ldots, w_{n}, z_{1}, \ldots, z_{n}\right)$ denote the canonical complex coordinates functions on $\mathbb{C}^{2 n+1}$. Recall that the projection 


$$
\begin{aligned}
& \pi: \mathbb{C}^{n+1} \times \mathbb{C}^{n}=\mathbb{C}^{2 n+1} \quad \rightarrow \quad \mathbb{C}^{n+1} \\
& (w, x)=\left(w_{0}, w_{1}, \ldots, w_{n}, z_{1}, \ldots, z_{n}\right) \longmapsto w=\left(w_{0}, w_{1}, \ldots, w_{n}\right)
\end{aligned}
$$

is called the front projection.

Then, for every holomorphic function $h: \mathbb{C}^{n} \rightarrow \mathbb{C}$, the map

$$
\begin{aligned}
i_{h}: \mathbb{C}^{n} & \longrightarrow \mathbb{C}^{n+1} \times \mathbb{C}^{n}=\mathbb{C}^{2 n+1} \\
z & \longmapsto\left(x_{h}(z), z\right)
\end{aligned}
$$

is a Legendrian immersion of $\mathbb{C}^{n}$ into $\left(\mathbb{C}^{2 n+1}, \omega\right)$ (that is, $i_{h}: \mathbb{C}^{n} \rightarrow \mathbb{C}^{2 n+1}$

is a holomorphic immersion, and $\left(T_{z} i_{h}\right)\left(\mathbb{C}^{n}\right) \subset \operatorname{Ker}\left[\omega_{i_{h}(z)}\right]$ for all $\left.z \in \mathbb{C}^{n}\right)$ of which $\mathcal{H}_{h}=x_{h}\left(\mathbb{C}^{n}\right)$ is the front $\left(\pi \circ i_{h}\right)\left(\mathbb{C}^{n}\right)$ in $\mathbb{C}^{n+1}$.

Indeed, for all $z=\left(z_{1}, \ldots, z_{n}\right) \in \mathbb{C}^{n}$ and $i \in\{1, \ldots, n\}$, we have

$$
\frac{\partial x_{h}}{\partial z_{i}}(z)=\left(-\sum_{j=1}^{n} z_{j} \frac{\partial^{2} h}{\partial z_{i} \partial z_{j}}(z), \frac{\partial^{2} h}{\partial z_{i} \partial z_{1}}(z), \ldots, \frac{\partial^{2} h}{\partial z_{i} \partial z_{n}}(z)\right)
$$

and hence

$$
\omega_{i_{h}(z)}\left(\frac{\partial x_{h}}{\partial z_{i}}(z), \frac{\partial I d_{\mathbb{C}^{n}}}{\partial z_{i}}(z)\right)=-\sum_{j=1}^{n} z_{j} \frac{\partial^{2} h}{\partial z_{i} \partial z_{j}}(z)+\sum_{j=1}^{n} z_{j} \frac{\partial^{2} h}{\partial z_{i} \partial z_{j}}(z)=0
$$

\subsection{Rational hedgehogs of the complex projective plane $P^{2}(\mathbb{C})$}

Here, we choose to work in the complex projective plane $P^{2}(\mathbb{C})$ equipped with the usual Fubini-Study Kähler form $\omega$ (see e.g. [2]). For any $\left(X_{1}, X_{2}, X_{3}\right) \in$ $\mathbb{C}^{3} \backslash\{0\},\left[X_{1}, X_{2}, X_{3}\right]$ will denote the homogeneous coordinates of the equivalent class of $\left(X_{1}, X_{2}, X_{3}\right)$ in $P^{2}(\mathbb{C})=\mathbb{C}^{3} / \mathbb{C}^{*}$.

Let $h: \mathbb{C} \rightarrow \mathbb{C}$ be a holomorphic map such that the projective curve $x_{h}$ : $\mathbb{C} \rightarrow P^{2}(\mathbb{C}), z \mapsto\left[x_{h}(z),-1\right]=\left[z h(z)-h^{\prime}(z), h^{\prime}(z),-1\right]$ satisfies

$$
\operatorname{Area}\left[x_{h}(\mathbb{C})\right]<+\infty .
$$

Then, the hedgehog curve $x_{h}: \mathbb{C} \rightarrow P^{2}(\mathbb{C})$ extends to a rational curve

$$
\begin{aligned}
x_{h}: & P^{1}(\mathbb{C}) \rightarrow P^{2}(\mathbb{C}) \\
& z \longmapsto x_{h}(z),
\end{aligned}
$$

which we call the rational hedgehog $\mathcal{H}_{h}:=x_{h}\left[P^{1}(\mathbb{C})\right]$ with support function

$$
h: P^{1}(\mathbb{C}) \rightarrow P^{1}(\mathbb{C}), z \longmapsto\left\{\begin{array}{l}
h(z) \quad \text { if } \quad z \in \mathbb{C} \\
\lim _{z \rightarrow \infty} h(z) \quad \text { if } \quad z=\infty .
\end{array}\right.
$$


Indeed Ahlfors lemma gives a description of rational curves as entire curves of bounded area $([3])$ :

"Let $X$ be a compact complex manifold and $f: \mathbb{C} \rightarrow X$ an entire curve (i.e. a non constant holomorphic map) such that Area $[f(\mathbb{C})]<+\infty$. Then $f$ extends to a holomorphic map from $P^{1}(\mathbb{C})$ to $X$, a rational curve".

\subsection{Evolute of a plane complex hedgehog as locus of its centers of curvature}

In classical differential geometry of curves, the evolute of a plane curve is the locus of all its centers of curvature or, equivalently, the envelope of its normal lines. In particular, the evolute of a plane hedgehog $\mathcal{H}_{h} \subset \mathbb{R}^{2}$ with support function $h \in C^{\infty}\left(\mathbb{S}^{1} ; \mathbb{R}\right)$ is the locus of all its centers of curvature $c_{h}(\theta):=$ $x_{h}(\theta)-R_{h}(\theta) u(\theta)$, where $R_{h}(\theta):=\operatorname{det}\left[T_{u(\theta)} x_{h}\right]=\left(h+h^{\prime \prime}\right)(\theta)$ is the socalled curvature function of $\mathcal{H}_{h}$, and $u(\theta):=(\cos \theta, \sin \theta),\left(\theta \in \mathbb{S}^{1}=\mathbb{R} / 2 \pi \mathbb{Z}\right)$. Equivalently, the evolute of $\mathcal{H}_{h}$ can be defined as the envelope of its 'normal lines' $N_{h}(\theta):=\left\{x_{h}(\theta)\right\}+\mathbb{R} u(\theta)$, that is, the hedgehog $\mathcal{H}_{\partial h}$ with support function $(\partial h)(\theta):=h^{\prime}\left(\theta-\frac{\pi}{2}\right)$. Note that in the hedgehog case, the centers of curvature $c_{h}(\theta)$ are well-defined for all $\theta \in \mathbb{S}^{1}$, even if $x_{h}^{\prime}(\theta)=R_{h}(\theta) u(\theta)$ is the null vector, since the curvature function $R_{h}(\theta)=\left(h+h^{\prime \prime}\right)(\theta)$ is well-defined for all $\theta \in \mathbb{S}^{1}$. Likewise, the normal line to $\mathcal{H}_{h}$ at $x_{h}(\theta)$ is well-defined, even if $x_{h}^{\prime}(\theta)=0$, as the perpendicular $N_{h}(\theta)$ to the support line $\langle x, u(\theta)\rangle=h(\theta)$ through the point $x_{h}(\theta)$. For plane real hedgehogs, it is convenient to keep in mind the following commutative diagram:

\begin{tabular}{|c|c|c|c|}
\hline \multicolumn{2}{|c|}{$\begin{aligned} \gamma_{h}: & \mathbb{S}^{1} \rightarrow P^{2}(\mathbb{R}) \\
& \theta \longmapsto[\cos \theta, \sin \theta, h(\theta)]\end{aligned}$} & $\begin{array}{c}\text { Projective duality } \\
\stackrel{*}{\leftrightarrow}\end{array}$ & $\begin{aligned} X_{h}: & \mathbb{S}^{1} \rightarrow \mathbb{R}^{2} \subset P^{2}(\mathbb{R}) \\
& \theta \longmapsto\left(x_{h}(\theta),-1\right)\end{aligned}$ \\
\hline & $\frac{d}{d \theta} \quad \downarrow \quad$ derivation & & $\downarrow \quad$ evolute \\
\hline$\gamma_{h}^{\prime}$ & $\begin{array}{l}\mathbb{S}^{1} \rightarrow P^{2}(\mathbb{R}) \\
\theta \longmapsto\left[-\sin \theta, \cos \theta, h^{\prime}(\theta)\right]\end{array}$ & $\stackrel{\text { Projective duality }}{\stackrel{*}{\leftrightarrow}}$ & $\begin{array}{l}\mathbb{S}^{1} \rightarrow \mathbb{R}^{2} \subset P^{2}(\mathbb{R}) \\
\theta \longmapsto\left(c_{h}(\theta),-1\right)\end{array}$ \\
\hline
\end{tabular}

where $c_{h}(\theta)=x_{\partial h}\left(\theta+\frac{\pi}{2}\right),\left(\theta \in \mathbb{S}^{1}\right)$. The main purpose of this subsection is to extend the notion of evolute to plane complex hedgehogs, together with its interpretation as locus of the centers of curvature. To this aim, we need to change our way of interpreting the transformation

$$
\begin{aligned}
\frac{d}{d \theta}: & \mathbb{S}^{1} \subset \mathbb{R}^{2} \quad \rightarrow \quad \mathbb{S}^{1} \subset \mathbb{R}^{2} \\
& u(\theta)=(\cos \theta, \sin \theta) \longmapsto u^{\prime}(\theta)=(-\sin \theta, \cos \theta)
\end{aligned}
$$

in the above diagram since we cannot consider the complex 'normal lines' to a complex hedgehog without antiholomorphic data being involved. Our choice is 
to identify $\mathbb{S}^{1}$ with the projective line $P^{1}(\mathbb{R})=\mathbb{R} \cup\{\infty\}$ and thus to consider the transformation

$$
\begin{aligned}
& P^{1}(\mathbb{R})=\mathbb{R} \cup\{\infty\} \quad \rightarrow \quad P^{1}(\mathbb{R})=\mathbb{R} \cup\{\infty\} \\
& {[\cos \theta, \sin \theta]=x \quad \mapsto \quad[-\sin \theta, \cos \theta]=\frac{-1}{x} .}
\end{aligned}
$$

In the case of complex hedgehogs, it is thus the following transformation which will play the same role:

$$
\begin{gathered}
P^{1}(\mathbb{C})=\mathbb{C} \cup\{\infty\} \quad \rightarrow \quad P^{1}(\mathbb{C})=\mathbb{C} \cup\{\infty\} \\
{[1, z]=z \quad \mapsto \quad[z,-1]=\frac{-1}{z}}
\end{gathered}
$$

In other words, we are going to consider the envelope of the family $\left(L_{h}^{\prime}(z)\right)_{z \in \mathbb{C}}$ of complex lines of $\mathbb{C}^{2}$ given by $L_{h}^{\prime}(z):=\left\{x_{h}(z)\right\}+\mathbb{C}(z,-1)$. For all $z \in \mathbb{C}$, $L_{h}^{\prime}(z)$ can be completed into a projective line $\widehat{L_{h}^{\prime}(z)}$ of $P^{2}(\mathbb{C})$ with equation

$$
z X_{1}-X_{2}+\left(z h(z)-\left(1+z^{2}\right) h^{\prime}(z)\right) X_{3}=0,
$$

where $\left[X_{1}, X_{2}, X_{3}\right]$ denote the homogeneous coordinates of the equivalent class of $\left(X_{1}, X_{2}, X_{3}\right) \in \mathbb{C}^{3} \backslash\{0\}$ in $P^{2}(\mathbb{C})$. Now, by projective duality, this family of projective lines $\left(\widehat{L_{h}^{\prime}(z)}\right)_{z \in \mathbb{C}}$ corresponds to the complex curve that is parametrized by

$$
\begin{array}{ccc}
\mathbb{C} & \rightarrow & P^{2}(\mathbb{C}) \\
z & \longmapsto & {\left[z,-1, z h(z)-\left(1+z^{2}\right) h^{\prime}(z)\right] .}
\end{array}
$$

\begin{tabular}{|c|c|c|}
\hline$\gamma_{h}: z \mapsto[1, z, h(z)]$ & $\begin{array}{c}\text { Projective duality } \\
\stackrel{*}{\leftrightarrow}\end{array}$ & $X_{h}: z \mapsto\left[x_{h}(z),-1\right]$ \\
\hline$\downarrow$ & & $\partial \quad \downarrow \quad$ evolute \\
\hline$\gamma_{\partial h}: w=\frac{-1}{z} \mapsto[1, w,(\partial h)(w)]$ & $\stackrel{\text { Projective duality }}{\stackrel{*}{\leftrightarrow}}$ & $\left(c_{h},-1\right): z \mapsto\left[x_{\partial h}\left(\frac{-1}{z}\right),-1\right]$ \\
\hline
\end{tabular}

Note that for $z \neq 0$, we have

$$
\left[z,-1, z h(z)-\left(1+z^{2}\right) h^{\prime}(z)\right]=[1, w,(\partial h)(w)],
$$

where $w=\frac{-1}{z}$ and $(\partial h)(w):=h\left(\frac{-1}{w}\right)+\left(w+\frac{1}{w}\right) h^{\prime}\left(\frac{-1}{w}\right)$.

Therefore, we have the following commutative diagram:

where $c_{h}(z):=x_{\partial h}\left(\frac{-1}{z}\right)=x_{h}(z)-\left(1+z^{2}\right) h^{\prime \prime}(z)(1, z)$. This expression of $c_{h}(z)$ has to be compared to the one giving the expression of the center of curvature of a real hedgehog $\mathcal{H}_{h}$ at a point $x_{h}(\theta): c_{h}(\theta)=x_{h}(\theta)-R_{h}(\theta) u(\theta)$, where $R_{h}$ is the curvature function of $\mathcal{H}_{h} \subset \mathbb{R}^{2}$. We shall see below that $c_{h}(z):=x_{\partial h}\left(\frac{-1}{z}\right)=x_{h}(z)-\left(1+z^{2}\right) h^{\prime \prime}(z)(1, z)$ can actually be interpreted as the center of curvature of the complex hedgehog $\mathcal{H}_{h}$ at the point $x_{h}(z)$. 
Definition 2 Let $h: \mathbb{C} \rightarrow \mathbb{C}$ be a holomorphic function. We shall say that the complex hedgehog with support function $(\partial h)(z)=h\left(\frac{-1}{z}\right)+\left(z+\frac{1}{z}\right) h^{\prime}\left(\frac{-1}{z}\right)$ is the evolute of the complex hedgehog $\mathcal{H}_{h}$.

Fundamental examples. If $h$ is the holomorphic function defined on the open $\operatorname{disc} D:=\{z \in \mathbb{C}|| z \mid<1\}$ by $h(z)=a_{1} z+a_{0}+\rho \sqrt{1+z^{2}}$, where $\left(a_{0}, a_{1}, \rho\right) \in \mathbb{C}^{3}$, then the complex hedgehog $\mathcal{H}_{h}=x_{h}(D)$ is reduced to the point $\left\{\left(a_{0}, a_{1}\right)\right\}$ if $\rho=0$, and it lies on the complex circle $\mathcal{C}\left(\left(a_{0}, a_{1}\right) ; \rho\right) \subset \mathbb{C}^{2}$ with equation $\left(X_{1}-a_{0}\right)^{2}+\left(X_{2}-a_{1}\right)^{2}=\rho^{2}$ if $\rho \neq 0$. In both cases, the evolute $\mathcal{H}_{\partial h}=c_{h}(D)$ is reduced to the point $\left\{\left(a_{0}, a_{1}\right)\right\}$. Indeed, for all $z \in \mathbb{C}, x_{h}(z)=\left(x_{h}^{1}(z), x_{h}^{2}(z)\right)=$ $\left(h(z)-z h^{\prime}(z), h^{\prime}(z)\right)$ is such that

$$
\left(x_{h}^{1}(z), x_{h}^{2}(z)\right)=\left(a_{0}+\rho \sqrt{1+z^{2}}-z \frac{z}{\sqrt{1+z^{2}}}, a_{1+} \frac{\rho z}{\sqrt{1+z^{2}}}\right)=\left(a_{0}, a_{1}\right)+\rho \frac{(1, z)}{\sqrt{1+z^{2}}}
$$

and

$c_{h}(z)=x_{h}(z)-\left(1+z^{2}\right) h^{\prime \prime}(z)(1, z)=x_{h}(z)-\left(1+z^{2}\right) \frac{\rho}{\left(1+z^{2}\right)^{\frac{3}{2}}} \frac{(1, z)}{\sqrt{1+z^{2}}}=\left(a_{0}, a_{1}\right)$.

More generally, let us replace $h: D \rightarrow \mathbb{C}, z \mapsto a_{1} z+a_{0}+\rho \sqrt{1+z^{2}}$ by any holomorphic function of the form $h: \mathcal{U} \rightarrow \mathbb{C}, z \mapsto a_{1} z+a_{0}+\rho q(z)$, where $\mathcal{U}$ is a connected open subset of $\mathbb{C} \backslash\{-i, i\}$, and $q(z)$ is the support function of the complex unit circle $\mathcal{C}((0,0) ; 1)$ in the neighbor of $z$, that is:

$$
q(z)=\left\{\begin{array}{l}
\sqrt{1+z^{2}} \quad \text { if } \quad|z|<1 \\
z \sqrt{1+\left(\frac{1}{z}\right)^{2}} \text { if }|z|>1 \\
\frac{z+\varepsilon}{\sqrt{2} \sqrt{1+\left(\frac{z-\varepsilon}{z+\varepsilon}\right)^{2}}} \text { if } \operatorname{sign}[\operatorname{Re}(z)]=\varepsilon \in\{-1,1\} .
\end{array}\right.
$$

We leave it to the reader to check that : $(i)$ the complex hedgehog $\mathcal{H}_{h}=$ $x_{h}(\mathcal{U})$ is reduced to the point $\left\{\left(a_{0}, a_{1}\right)\right\}$ if $\rho=0$, and it lies on the complex circle $\mathcal{C}\left(\left(a_{0}, a_{1}\right) ; \rho\right)$ with equation $\left(X_{1}-a_{0}\right)^{2}+\left(X_{2}-a_{1}\right)^{2}=\rho^{2}$ if $\rho \neq 0 ;$ (ii) moreover, in both cases, the evolute $\mathcal{H}_{\partial h}=c_{h}(\mathcal{U})$ is reduced to the point $\left\{\left(a_{0}, a_{1}\right)\right\}$.

Definition 3 Let $\mathcal{H}_{f}$ and $\mathcal{H}_{g}$ be two complex hedgehogs in $\mathbb{C}^{2}$, and let $z_{0} \in \mathbb{C}$ be such that $x_{f}\left(z_{0}\right)=x_{g}\left(z_{0}\right)$. We shall say that $\mathcal{H}_{f}$ and $\mathcal{H}_{g}$ have a contact of order $\geq 2$ at $x_{f}\left(z_{0}\right)=x_{g}\left(z_{0}\right)$, if: $\forall m \in\{0,1,2\}, f^{(m)}\left(z_{0}\right)=g^{(m)}\left(z_{0}\right)$.

Given any complex hedgehog with holomorphic support function $h: \mathcal{U} \rightarrow \mathbb{C}$, where $\mathcal{U}$ is any connected open subset of $\mathbb{C} \backslash\{-i, i\}$, a straightforward computation shows that, for any $z_{0} \in \mathcal{U}$, the hedgehog with support function

$$
c: \mathcal{U} \rightarrow \mathbb{C}, z \mapsto c_{h}(z):=c_{h}^{2}\left(z_{0}\right) z+c_{h}^{1}\left(z_{0}\right)+q\left(z_{0}\right)^{3} h^{\prime \prime}\left(z_{0}\right) q(z),
$$


(which is reduced to the point $\left\{c_{h}\left(z_{0}\right)\right\}$ if $h^{\prime \prime}\left(z_{0}\right)=0$, or which lies on the complex circle with equation $\left(X_{1}-c_{h}^{1}\left(z_{0}\right)\right)^{2}+\left(X_{2}-c_{h}^{2}\left(z_{0}\right)\right)^{2}=q\left(z_{0}\right)^{6} h^{\prime \prime}\left(z_{0}\right)^{2}$ if $\left.h^{\prime \prime}\left(z_{0}\right) \neq 0\right)$, has a contact of order $\geq 2$ with $\mathcal{H}_{h}=x_{h}(\mathcal{U})$ at $x_{h}\left(z_{0}\right)$.

Definition 4 Let $h: \mathcal{U} \rightarrow \mathbb{C}$ be a holomorphic function where $\mathcal{U}$ is a connected subset of $\mathbb{C} \backslash\{-i, i\}$. For any $z_{0} \in \mathcal{U}$, we shall say that $c_{h}\left(z_{0}\right)$ is the center of curvature of $\mathcal{H}_{h}=x_{h}(\mathcal{U})$ at $x_{h}\left(z_{0}\right)$, and, if $z_{0} \in \mathcal{U}$ is a regular point of $x_{h}: \mathcal{U} \rightarrow \mathbb{C}^{2}$ (that is, if $\left.h^{\prime \prime}\left(z_{0}\right) \neq 0\right)$, we shall say that the complex circle with equation

$$
\left(X_{1}-c_{h}^{1}\left(z_{0}\right)\right)^{2}+\left(X_{2}-c_{h}^{2}\left(z_{0}\right)\right)^{2}=q\left(z_{0}\right)^{6} h^{\prime \prime}\left(z_{0}\right)^{2}
$$

is the osculating complex circle of $\mathcal{H}_{h}$ at $x_{h}\left(z_{0}\right)$.

Naturally, we define the complex curvature function of a hedgehog $\mathcal{H}_{h}=$ $x_{h}(\mathcal{U})$ as follows.

Definition 5 Let $h: \mathcal{U} \rightarrow \mathbb{C}$ be a holomorphic function where $\mathcal{U}$ is a connected subset of $\mathbb{C} \backslash\{-i, i\}$. We define the curvature function of $\mathcal{H}_{h}=x_{h}(\mathcal{U})$ to be the function $R_{h}: \mathcal{U} \rightarrow \mathbb{C}$ that is given by $R_{h}(z):=q(z)^{3} h^{\prime \prime}(z)$ for all $z \in \mathcal{U}$.

Thus, for any $z \in \mathcal{U}$, the center of curvature of $\mathcal{H}_{h}=x_{h}(\mathcal{U})$ at $x_{h}(z)$ can be expressed as follows:

$$
c_{h}(z)=x_{h}(z)-R_{h}(z) u(z),
$$

where

$$
u(z):=\frac{1+z^{2}}{q(z)^{3}}(1, z) \in \mathcal{C}((0,0) ; 1) .
$$

Of course, this expression of $c_{h}(z)$ has to be compared to the one giving the expression of the center of curvature of a real hedgehog $\mathcal{H}_{h}$ at a point $x_{h}(\theta)$ : $c_{h}(\theta)=x_{h}(\theta)-R_{h}(\theta) u(\theta)$, where $R_{h}$ is the curvature function of $\mathcal{H}_{h} \subset \mathbb{R}^{2}$.

Remark. With our definitions, the complex circle $\mathcal{C}\left(\left(a_{0}, a_{1}\right) ; \rho\right) \subset \mathbb{C}^{2}$ with equation $\left(X_{1}-a_{0}\right)^{2}+\left(X_{2}-a_{1}\right)^{2}=\rho^{2}$, where $\left(a_{0}, a_{1}, \rho\right) \in \mathbb{C}^{2} \times \mathbb{C}^{*}$, can be locally regarded as a hedgehog with radius of curvature equal to $\rho$ (possibly after a suitable chart change on the Riemann sphere).

\subsection{Real and imaginary parts of $\mathcal{H}_{h} \subset \mathbb{C}^{2}$ regarded as hedgehogs of $\mathbb{R}^{3}$}

We know that if $f$ and $g$ are taken to be the real and imaginary parts respectively of a holomorphic function $h: \mathbb{C} \rightarrow \mathbb{C}, z=x+i y \mapsto h(z)=f(x, y)+i g(x, y)$, then $f$ and $g$ are harmonic functions satisfying the Cauchy-Riemann equations, that is,

$$
\frac{\partial f}{\partial x}(x, y)=\frac{\partial g}{\partial y}(x, y) \quad \text { and } \quad \frac{\partial f}{\partial y}(x, y)=-\frac{\partial g}{\partial x}(x, y)
$$


for all $(x, y) \in \mathbb{R}^{2}$. The aim of this subsection is to show that, in this context, $f$ and $g$ determine two hedgehogs $\mathcal{H}_{F}$ and $\mathcal{H}_{G}$ of $\mathbb{R}^{3}$ that can be regarded globally as the orthogonal projections of the complex hedgehog $\mathcal{H}_{h}$ of $\mathbb{C}^{2} \cong \mathbb{R}^{4}$ into $e_{2}^{\perp}$ and $e_{1}^{\perp}$ respectively, where $\left(e_{1}, e_{2}, e_{3}, e_{4}\right)$ is the canonical basis of $\mathbb{R}^{4}$ and where $e_{i}^{\perp}$ denotes the 3 -dimensional subspace of $\mathbb{R}^{4}$ that is orthogonal to $e_{i}(1 \leq i \leq 4)$. These hedgehogs $\mathcal{H}_{F}$ and $\mathcal{H}_{G}$ of $\mathbb{R}^{3}$ will be modeled on the hemisphere $\mathbb{S}_{+}^{2}$ of $\mathbb{S}^{2} \subset \mathbb{R} \times \mathbb{C}$ that is contained in $\mathbb{R}_{+}^{*} \times \mathbb{C}$. To any $z \in \mathbb{C}$ we associate the point $\nu(z):=(1, z) / \sqrt{1+|z|^{2}}$ of $\mathbb{S}_{+}^{2}$. The orthogonal projection map from $\mathbb{C}^{2} \cong \mathbb{R}^{4}$ onto $e_{i}^{\perp}$ will be denoted by $\pi_{e_{i}^{\perp}}$.

Proposition 6 Let $h: \mathbb{C} \rightarrow \mathbb{C}$ be a holomorphic function the real and imaginary parts of which are $f$ and $g$ respectively:

$$
h(x+i y)=f(x, y)+i g(x, y) \quad \text { for all }(x, y) \in \mathbb{R}^{2} .
$$

We have then

$$
\pi_{e_{2}^{\perp}}\left[x_{h}(\bar{z})\right]=x_{F}(\nu(z)) \quad \text { and } \quad \pi_{e_{1}^{\perp}}\left[x_{h}(i \bar{z})\right]=x_{G}(\nu(z)),
$$

where $F$ and $G$ are respectively defined by:

$$
F(\nu(z))=\frac{\operatorname{Re}(h(\bar{z}))}{\sqrt{1+|z|^{2}}} \text { and } G(\nu(z))=\frac{\operatorname{Im}(h(i \bar{z}))}{\sqrt{1+|z|^{2}}}
$$

We shall of course say that the hedgehogs $\mathcal{H}_{F}$ and $\mathcal{H}_{G}$ are the real and imaginary hedgehog parts of $\mathcal{H}_{h}$.

Proof. We first note that an easy computation making use of the CauchyRiemann equations gives:

$$
\begin{aligned}
x_{h}(z) & =\left(x_{1}(z)+i y_{1}(z), x_{2}(z)+i y_{2}(z)\right) \in \mathbb{C}^{2} \\
& \cong\left(x_{1}(z), y_{1}(z), x_{2}(z), y_{2}(z)\right) \in \mathbb{R}^{4},
\end{aligned}
$$

where

$$
\left\{\begin{array}{l}
x_{1}(z)=f(x, y)-x \frac{\partial f}{\partial x}(x, y)-y \frac{\partial f}{\partial y}(x, y) \\
y_{1}(z)=g(x, y)-x \frac{\partial g}{\partial x}(x, y)-y \frac{\partial g}{\partial y}(x, y) \\
x_{2}(z)=\frac{\partial f}{\partial x}(x, y)=\frac{\partial g}{\partial y}(x, y) \\
y_{2}(z)=-\frac{\partial f}{\partial y}(x, y)=\frac{\partial g}{\partial x}(x, y),
\end{array}\right.
$$

for all $z=x+i y,\left((x, y) \in \mathbb{R}^{2}\right)$.

Next, we consider the positively 1-homogeneous function $F: \mathbb{R}_{+}^{*} \times \mathbb{R}^{2} \rightarrow \mathbb{R}$ given by

$$
F(X, Y, Z):=X f\left(\frac{Y}{X},-\frac{Z}{X}\right) \quad \text { for all }(X, Y, Z) \in \mathbb{R}_{+}^{*} \times \mathbb{R}^{2} .
$$


A straightforward computation then shows that the Euclidean gradient of $F$ is given by

$$
\begin{aligned}
\nabla F(X, Y, Z) & =\left(f\left(\frac{Y}{X},-\frac{Z}{X}\right)-\frac{Y}{X} \frac{\partial f}{\partial x}\left(\frac{Y}{X},-\frac{Z}{X}\right)+\frac{Z}{X} \frac{\partial f}{\partial y}\left(\frac{Y}{X},-\frac{Z}{X}\right),\right. \\
& \frac{\partial f}{\partial x}\left(\frac{Y}{X},-\frac{Z}{X}\right) \\
& \left.-\frac{\partial f}{\partial y}\left(\frac{Y}{X},-\frac{Z}{X}\right)\right)
\end{aligned}
$$

for all $(X, Y, Z) \in \mathbb{R}_{+}^{*} \times \mathbb{R}^{2}$. Thus,

$$
\begin{aligned}
x_{F}(\nu(z)) & =\nabla F(\nu(z)) \\
& =\left(f(x,-y)-x \frac{\partial f}{\partial x}(x,-y)-(-y) \frac{\partial f}{\partial y}(x,-y), \frac{\partial f}{\partial x}(x,-y),-\frac{\partial f}{\partial y}(x,-y)\right) \\
& =\left(x_{1}(\bar{z}), x_{2}(\bar{z}), y_{2}(\bar{z})\right)=\pi_{e_{2}^{\perp}}\left[x_{h}(\bar{z})\right],
\end{aligned}
$$

for all $z=x+i y,\left((x, y) \in \mathbb{R}^{2}\right)$. In the same manner, we can easily check that

$$
x_{F}(\nu(z))=\left(y_{1}(i \bar{z}), x_{2}(i \bar{z}), y_{2}(i \bar{z})\right)=\pi_{e_{1}^{\perp}}\left[x_{h}(i \bar{z})\right] \quad \text { for all } z \in \mathbb{C} .
$$

\section{Towards a Brunn-Minkowski theory for com- plex hedgehogs}

As already mentioned above, the notion of a hedgehog curve or surface was born from the study of the Brunn-Minkowski theory. It is therefore permissible to think that the development of a 'theory of mixed volumes for complex hedgehogs' (replacing Euclidean volumes by symplectic ones) might be a promising way of research. In this section, we will just mention first two observations.

\subsection{Mixed symplectic area}

Let $\mathbb{C}^{2}$ be the complex Euclidean space endowed with the standard Hermitian inner product $\langle., .\rangle_{\mathbb{C}^{2}}$. We are interested in the symplectic area of complex hedgehogs in this Kähler manifold $\left(\mathbb{C}^{2}, J, \omega\right)$, where $J$ is the complex structure and $\omega$ the 2 -form $\omega(X, Y):=\operatorname{Re}\left(\langle J X, Y\rangle_{\mathbb{C}^{2}}\right)$. Any nontrivial complex hedgehog of $\mathbb{C}^{2}$ modeled on the unit open disk $\mathbb{D}$ of $\mathbb{C}$ is a holomorphic curve (i.e. a nonconstant map from the complex plane to $\mathbb{C}^{2}$ ). Now, it is well-known that the Riemannian area of holomorphic curves is equal to their symplectic area, and hence that holomorphic curves have positive area (the reader that is not familiar with holomorphic curves can find details in Subsection 1.1 of [17] ). An immediate consequence is the following result, which has to be compared to classical geometric inequalities for convex bodies (see [16, p. 369 and p. 382]). 
Theorem 7 Let $\mathcal{H}(\mathbb{D})$ be the complex linear space of holomorphic functions $h: \mathbb{D} \rightarrow \mathbb{C}$ defined up to a similitude and consider

$$
\operatorname{Area}\left[x_{h}(\mathbb{D})\right]:=\int_{x_{h}(\mathbb{D})} \omega .
$$

Then the map $\sqrt{A}: \mathcal{H}(\mathbb{D}) \rightarrow \mathbb{R}_{+}, h \mapsto \sqrt{\text { Area }\left[x_{h}(\mathbb{D})\right]}$ is a norm associated with a scalar product $(h, k) \longmapsto A(h, k)$, which can be interpreted as a mixed symplectic area. In particular, for any $(h, k) \in \mathcal{H}(\mathbb{D})^{2}$, we have

$$
\sqrt{A(h+k)} \leq \sqrt{A(h)}+\sqrt{A(k)}
$$

and

$$
A(h, k)^{2} \leq A(h) A(k),
$$

with equalities if, and only if, $\mathcal{H}_{h}$ and $\mathcal{H}_{k}$ are homothetic (here, "homothetic" means that there exists $(\lambda, \mu) \in \mathbb{R}^{2}-\{(0,0)\}$ such that $\left.\lambda h+\mu k=0\right)$.

\subsection{A sharp estimation of the area using the energy}

Note that we have the following sharp estimate of Area $\left[x_{h}(\mathbb{D})\right]$, which is better than that well-known for an arbitrary smooth loop $\gamma: \mathbb{S}^{1} \rightarrow V$ in a symplectic vector space $(V, \omega)$ (namely, $|A(\gamma)| \leq E(\gamma)$, see for instance [14, pp. 87-88]):

Theorem 8 Assume that $h: \mathbb{D} \rightarrow \mathbb{C}$ is the sum of a power series $\sum h_{n} z^{n}$ with radius of convergence $R>1$ :

$$
h(z)=\sum_{n=0}^{+\infty} h_{n} z^{n} \quad \text { for all } z \in \mathbb{D} .
$$

Then

$$
\text { Area }\left[x_{h}(\mathbb{D})\right] \leq \frac{3}{4} E\left(x_{h}\right),
$$

where $E\left(x_{h}\right)$ is the energy of the loop $x_{h}: \mathbb{S}^{1}=\mathbb{R} / 2 \pi \mathbb{Z} \rightarrow \mathbb{C}^{2}, \theta \mapsto x_{h}\left(e^{i \theta}\right)$, that is:

$$
E\left(x_{h}\right):=\frac{1}{2} \int_{0}^{2 \pi}\left|\frac{d}{d \theta}\left[x_{h}\left(e^{i \theta}\right)\right]\right|^{2} d \theta .
$$

Furthermore, the equality holds if, and only if, the function $h$ is of the form $h(z)=a_{m} z^{m}+a_{1} z+a_{0}$, where $m \in \mathbb{N}$ and $\left(a_{0}, a_{1}, a_{m}\right) \in \mathbb{C}^{3}$.

Proof. Consider the Fourier expansion of $H(\theta):=h\left(e^{i \theta}\right)$ on $\mathbb{S}^{1}=\mathbb{R} / 2 \pi \mathbb{Z}$ :

$$
H(\theta):=\sum_{n=0}^{+\infty} h_{n} e^{i n \theta}
$$


An easy computation immediately gives:

$$
\forall \theta \in \mathbb{S}^{1}, \quad x_{h}(\theta):=\sum_{n=0}^{+\infty} e^{i n \theta}\left((1-n) h_{n},(n+1) h_{n+1}\right) .
$$

Using the formula known for the action $A(\gamma):=(1 / 2) \int_{0}^{2 \pi} \omega\left(\gamma(\theta), \gamma^{\prime}(\theta)\right) d \theta$ of an arbitrary smooth loop $\gamma: \mathbb{S}^{1} \rightarrow \mathbb{C}^{2}$ (see e.g. at the top of the page 88 in [14]), we then deduce that:

$$
\text { Area }\left[x_{h}(\mathbb{D})\right]=-A\left(x_{h}\right)=\pi \sum_{n=0}^{+\infty} n\left((n-1)^{2}\left|h_{n}\right|^{2}+(n+1)^{2}\left|h_{n+1}\right|^{2}\right) .
$$

Separating into two sums and re-indexing in the first one, we then obtain:

$$
\text { Area }\left[x_{h}(\mathbb{D})\right]=\pi \sum_{n=1}^{+\infty} n(n+1)(2 n+1)\left|h_{n+1}\right|^{2}=6 \pi \sum_{n=1}^{+\infty}\left(\sum_{k=1}^{n} k^{2}\right)\left|h_{n+1}\right|^{2} .
$$

On the other hand, we have: $\forall \theta \in \mathbb{S}^{1}$,

$$
\frac{d}{d \theta}\left[x_{h}\left(e^{i \theta}\right)\right]=e^{-i \theta}\left(H^{\prime}(\theta)+i H^{\prime \prime}(\theta)\right)\left(e^{i \theta},-1\right)
$$

and hence

$$
\left|\frac{d}{d \theta}\left[x_{h}\left(e^{i \theta}\right)\right]\right|^{2}=2\left|H^{\prime}+i H^{\prime \prime}\right|(\theta)^{2}=2\left|H^{\prime \prime}-i H^{\prime}\right|(\theta)^{2}=2\left|h^{\prime \prime}\left(e^{i \theta}\right)\right|^{2} .
$$

Therefore Parseval's identity yields:

$E\left(x_{h}\right):=\frac{1}{2} \int_{0}^{2 \pi}\left|\frac{d}{d \theta}\left[x_{h}\left(e^{i \theta}\right)\right]\right|^{2} d \theta=\int_{0}^{2 \pi}\left|h^{\prime \prime}\left(e^{i \theta}\right)\right|^{2} d \theta=8 \pi \sum_{n=1}^{+\infty}\left(\sum_{k=1}^{n} k\right)^{2}\left|h_{n+1}\right|^{2}$,

since

$$
h^{\prime \prime}(z)=\sum_{n=1}^{+\infty} n(n+1) h_{n+1} z^{n-1}=2 \sum_{n=1}^{+\infty}\left(\sum_{k=1}^{n} k\right) h_{n+1} z^{n-1} .
$$

This completes the proof.

\section{Real hedgehogs in $\mathbb{C}^{n} \cong \mathbb{R}^{2 n}$ and their evolutes}

In the Euclidean plane, the evolute of a hedgehog is the locus of all its centers of curvature or, equivalently, the envelope of its normal lines. In order to find an analogue in any even higher dimension, we make use of the following trick. First, we fix a linear complex structure $J$ on $\mathbb{R}^{2 n}$ (that is, an endomorphism $J$ of $\mathbb{R}^{2 n}$ such that $\left.J^{2}=-I d_{\mathbb{R}^{2 n}}\right)$. Given any hedgehog with smooth support function $h$ in $\mathbb{R}^{2 n}$, we then define the normal hyperplane to $\mathcal{H}_{h}$ at a point $x_{h}(u)$, say $N_{h}(u)$, as the affine hyperplane $\left\{x_{h}(u)\right\}+J\left(u^{\perp}\right)$, where $u^{\perp}$ is the $(2 n-1)$-dimensional subspace of $\mathbb{R}^{2 n}$ that is orthogonal to $u$. Finally, we define the evolute of $\mathcal{H}_{h}$ in $\left(\mathbb{R}^{2 n}, J\right)$ as the envelope of the family of normal hyperplanes $\left(N_{h}(u)\right)_{u \in \mathbb{S}^{2 n-1}}$ in $\mathbb{R}^{2 n}$. Let us begin by considering carefully the four dimensional case. 


\subsection{Evolutes of hedgehogs hypersurfaces in $\mathbb{R}^{4}$}

In what follows, we identify $\mathbb{R}^{4}$ with the quaternion algebra $\mathbb{H}$ and thus the unit sphere $\mathbb{S}^{3}$ with the set $\mathbb{S}_{\mathbb{H}}^{1}$ of unit quaternions. To any pure unit quaternion $v$, we associate the linear complex structure $J_{v}: \mathbb{R}^{4} \rightarrow \mathbb{R}^{4}, x \longmapsto v x$. We denote by $\omega_{v}$ the associated Kähler form (i.e. the alternating 2-form $\omega_{v}(X, Y)=\left\langle J_{v} X, Y\right\rangle$, where $\langle.,$.$\left.\rangle is the standard Euclidean metric on \mathbb{R}^{4}\right)$. Recall that we can retrieve $\langle.,$.$\rangle from \omega_{v}:\langle X, Y\rangle=\omega_{v}\left(X, J_{v} Y\right)$. Particularizing our definition of evolute hedgehogs to the four dimensional case, we get the following definition.

Definition 9 Let $h \in C^{\infty}\left(\mathbb{S}^{3} ; \mathbb{R}\right)$. We define the evolute of $\mathcal{H}_{h}$ in the Kähler vector space $\left(\mathbb{R}^{4}, J_{v}, \omega_{v}\right)$ to be the envelope of the family of normal hyperplanes $\left(N_{h}^{v}(u)\right)_{u \in \mathbb{S}^{3}}$ with equation

$$
\left\langle x-x_{h}(u), J_{v}(u)\right\rangle=0 .
$$

Proposition 10 Let $h \in C^{\infty}\left(\mathbb{S}^{3} ; \mathbb{R}\right)$. The evolute of $\mathcal{H}_{h}$ in $\left(\mathbb{R}^{4}, J_{v}, \omega_{v}\right)$ is the hedgehog $\mathcal{H}_{\partial_{v} h}$ with support function

$$
\partial_{v} h: \mathbb{S}^{3} \rightarrow \mathbb{R}, u \longmapsto\left\langle\nabla h\left(-J_{v}(u)\right), u\right\rangle,
$$

where $\langle.,$.$\rangle is the standard Euclidean metric on \mathbb{R}^{4}$, and $\nabla h$ the gradient of $h$.

Proof. Since $J_{v}: \mathbb{R}^{4} \rightarrow \mathbb{R}^{4}$ is an isometry such that $J_{v}^{2}=-I d_{\mathbb{R}^{4}}$, the evolute of $\mathcal{H}_{h}$ in $\left(\mathbb{R}^{4}, J_{v}, \omega_{v}\right)$ can be regarded as the envelope of the family of hyperplanes $\left(N_{h}^{v}\left(-J_{v}(u)\right)\right)_{u \in \mathbb{S}^{3}}$ with equation

$$
\left\langle x-x_{h}\left(-J_{v}(u)\right), u\right\rangle=0,
$$

that is, as the hedgehog $\mathcal{H}_{\partial_{v} h}$ of $\mathbb{R}^{4}$ with support function

$$
\partial_{v} h(u)=\left\langle x_{h}\left(-J_{v}(u)\right), u\right\rangle=\left\langle\nabla h\left(-J_{v}(u)\right), u\right\rangle \text {. }
$$

Remark 11 By abuse of language, the hedgehog with support function $\partial_{v} h$ will also be called 'the evolute of $\mathcal{H}_{h}$ with respect to (the pure unit) quaternion $v$ '.

\section{Parametrization of the evolute of $\mathcal{H}_{\partial_{v} h}$ and interpretation}

It follows immediately from definitions that $x_{\partial_{v} h}: \mathbb{S}^{3} \rightarrow \mathbb{R}^{4}$ associates with each $u \in \mathbb{S}^{3}$ the unique solution of the system

$$
\left\{\begin{aligned}
&\left\langle x, J_{v}(u)\right\rangle=\left\langle x_{h}(u), J_{v}(u)\right\rangle \\
& \forall X \in T_{u} \mathbb{S}^{3},\left\langle x, J_{v}(X)\right\rangle=\left\langle T_{u} x_{h}(X), J_{v}(u)\right\rangle+\left\langle x_{h}(u), J_{v}(X)\right\rangle,
\end{aligned}\right.
$$

which is equivalent to 


$$
\left\{\begin{array}{l}
\left\langle x-x_{h}(u), J_{v}(u)\right\rangle=0 \\
\forall X \in T_{u} \mathbb{S}^{3},\left\langle x-x_{h}(u), J_{v}(X)\right\rangle=\left\langle J_{v}\left(T_{u} x_{h}\left(J_{v}(u)\right)\right), J_{v}(X)\right\rangle,
\end{array}\right.
$$

because $\left\langle T_{u} x_{h}(X), J_{v}(u)\right\rangle=\left\langle T_{u} x_{h}\left(J_{v}(u)\right), X\right\rangle=\left\langle J_{v}\left(T_{u} x_{h}\left(J_{v}(u)\right)\right), J_{v}(X)\right\rangle$ since $T_{u} x_{h}$ is a symmetric endomorphism of $T_{u} \mathbb{S}^{3}$ and $J_{v}$ an isometry of $\mathbb{R}^{4}$. Therefore:

$$
\forall u \in \mathbb{S}^{3}, \quad x_{\partial_{v} h}(u)=x_{h}(u)+J_{v}\left(T_{u} x_{h}\left(J_{v}(u)\right)\right)=x_{h}(u)+v T_{u} x_{h}\left(J_{v}(u)\right) .
$$

In other words, we have the following.

Proposition 12 Let $h \in C^{\infty}\left(\mathbb{S}^{3} ; \mathbb{R}\right)$ and let $v$ be a pure unit quaternion. For all $u \in \mathbb{S}^{3}$,

$$
x_{\partial_{v} h}(u)=x_{h}(u)-R_{h}(u, v) u,
$$

where $R_{h}(u, v):=-v T_{u} x_{h}\left(J_{v}(u)\right) \bar{u}$; here $\bar{u}$ of course refers to the quaternion conjugate of $u$.

\section{Comparison to the planar case and interpretation}

This expression of $x_{\partial_{v} h}(u)$ has to be compared to the one of the center of curvature of a plane hedgehog $\mathcal{H}_{h}$ at a point $x_{h}(\theta)$ :

$$
c_{h}(\theta):=x_{h}(\theta)-R_{h}(\theta) u(\theta),
$$

where $R_{h}:=h+h^{\prime \prime}$ is the curvature function of $\mathcal{H}_{h}$. Identifying $\mathbb{R}^{2}$ to $\mathbb{C}$, and thus $T_{u_{\theta}} \mathbb{S}^{1}$ with $\mathbb{R}\left(i e^{i \theta}\right)$, this last formula can be rewritten as

$$
c_{h}\left(e^{i \theta}\right):=x_{h}\left(e^{i \theta}\right)-R_{h}\left(e^{i \theta}\right) e^{i \theta},
$$

where $R_{h}\left(e^{i \theta}\right):=-i T_{u_{\theta}} x_{h}\left(i e^{i \theta}\right) e^{-i \theta}$.

We shall see below that:

$R_{h}(., v): u_{\theta}:=(\cos \theta) u+(\sin \theta) v u \longmapsto R_{h}\left(u_{\theta}, v\right)$ can be interpreted as a quaternionic curvature function of $x_{h}\left(\mathbb{S}_{u, v}^{1}\right)$, where $\mathbb{S}_{u, v}^{1}$ denotes the unit circle of the vector plane $\mathbb{C}(u, v):=\mathbb{R} u+\mathbb{R} J_{v}(u)$ oriented by $\left(u, J_{v}(u)\right)$.

The reason why the map $\theta \longmapsto R_{h}\left(e^{i \theta}\right)$ is real for $h \in C^{\infty}\left(\mathbb{S}^{1} ; \mathbb{R}\right)$, whereas $u \mapsto R_{h}(u, v)$ is quaternionic for $h \in C^{\infty}\left(\mathbb{S}^{3} ; \mathbb{R}\right)$, is because the product of two purely imaginary complex numbers is a real number, whereas the product of two purely imaginary quaternions can have both nontrivial real and imaginary parts.

\section{Complement to the planar case}

We introduced "the" evolute $\mathcal{H}_{\partial h}$ of a plane hedgehog $\mathcal{H}_{h}$ as the envelope of its normal lines. But in fact there are two of them if we take into account the 
choice of coorientation of the normal line. Of course, we could have introduce evolutes of hedgehog curves in $\mathbb{R}^{2}$ in the same way as we have just done for evolutes of hedgehogs hypersurfaces in $\mathbb{R}^{4}$. Identifying $\mathbb{R}^{2}$ with the complex plane $\mathbb{C}$, we can associate to any $v \in\{-i, i\}$ the linear complex structure $J_{v}$ : $\mathbb{C} \rightarrow \mathbb{C}, x \longmapsto v x$ and the associated Kähler form $\omega_{v}(X, Y)=\left\langle J_{v} X, Y\right\rangle$, where $\langle.,$.$\rangle is the standard Euclidean metric on \mathbb{R}^{2}$, and then, define the evolute of the plane hedgehog with support function $h \in C^{\infty}\left(\mathbb{S}^{1} ; \mathbb{R}\right)$ in $\left(\mathbb{R}^{2}, J_{v}\right)$ to be the envelope $\mathcal{H}_{\partial_{v} h}$ of the family of normal lines $\left(N_{h}^{v}(u)\right)_{u \in \mathbb{S}^{1}}$ with equation

$$
\left\langle x-x_{h}(u), J_{v}(u)\right\rangle=0 .
$$

If we do so, we can immediately check that $\mathcal{H}_{\partial_{v} h}$ has support function

$$
\partial_{v} h: \mathbb{S}^{1} \rightarrow \mathbb{R}, u \longmapsto\left\langle\nabla h\left(-J_{v}(u)\right), u\right\rangle .
$$

In other words, $\left(\partial_{i} h\right)(\theta)=h^{\prime}(\theta-\pi / 2)$ and $\left(\partial_{-i} h\right)(\theta)=-h^{\prime}(\theta+\pi / 2)$ for all $\theta \in \mathbb{S}^{1}=\mathbb{R} / 2 \pi \mathbb{Z}$.

Note that, in the 2 or 4 -dimensional case, the evolutes $\mathcal{H}_{\partial_{v} h}$ and $\mathcal{H}_{\partial_{-v} h}$ are one and the same hypersurface of $\mathbb{R}^{2 n}(n=1,2)$ but corresponding to opposite coorientations of the normal hyperplanes of $\mathcal{H}_{h}$ :

$$
\left(\partial_{-v} h\right)(u)=\left\langle\nabla h\left(-J_{-v}(u)\right), u\right\rangle=-\left\langle\nabla h\left(-J_{v}(-u)\right),-u\right\rangle=-\left(\partial_{v} h\right)(-u) .
$$

\section{Geometrical interpretation of the Hodge Laplacian}

Taking the Hodge Laplacian of $h \in C^{\infty}\left(\mathbb{S}^{1} ; \mathbb{R}\right)$ is tantamount to taking the evolute in $\left(\mathbb{R}^{2}, J_{i}\right)$ of the evolute of $\mathcal{H}_{h}$ in $\left(\mathbb{R}^{2}, J_{-i}\right)$, or conversely, the evolute in $\left(\mathbb{R}^{2}, J_{-i}\right)$ of the evolute of $\mathcal{H}_{h}$ in $\left(\mathbb{R}^{2}, J_{i}\right)$. Indeed, for any $h \in C^{\infty}\left(\mathbb{S}^{1} ; \mathbb{R}\right)$, we have $\left(\partial_{i} \circ \partial_{-i}\right)(h)=\left(\partial_{-i} \circ \partial_{i}\right)(h)=-h^{\prime \prime}=\Delta h$, where $\Delta$ is the Hodge Laplacian on $\mathbb{S}^{1}$.

This result can be extended as follows to dimension 4. Let $h \in C^{\infty}\left(\mathbb{S}^{3} ; \mathbb{R}\right)$ and $u \in \mathbb{S}^{3}$. If $v$ is a pure unit quaternion such that $J_{v}(u)$ is an eigenvector of the Hessian $\left(\nabla^{2} h\right)_{u}$ of $h$ at $u$ corresponding to the eigenvalue $\lambda$, then:

$$
\begin{aligned}
\partial_{-v}\left(\partial_{v} h\right)(u) & =-\partial_{v}\left(\partial_{v} h\right)(-u)=-\partial_{v}^{2} h(-u)=-\partial_{v}^{2} h\left(J_{v}^{2}(u)\right) \\
& =-\left(\nabla^{2} h\right)_{u}\left(J_{v}(u), J_{v}(u)\right)=-\lambda .
\end{aligned}
$$

Therefore, if $v_{1}, v_{2}, v_{3}$ are pure unit quaternions such that $J_{v_{1}}(u), J_{v_{2}}(u), J_{v_{3}}(u)$ are eigenvectors of the Riemannian Hessian $\left(\nabla^{2} h\right)_{u}$, corresponding to eigenvalues $\lambda_{1}, \lambda_{2}, \lambda_{3}$, that form an orthonormal basis of $T_{u} \mathbb{S}^{3}$, then:

$$
\Delta h(u)=-\sum_{i=1}^{3} \lambda_{i}(u)=\sum_{i=1}^{3}\left(\partial_{-v_{i}} \circ \partial_{v_{i}}\right)(h)(u) .
$$




\section{Decomposition of hedgehogs into sums of remarkable pedal hyper- surfaces}

Let $(v, w)$ be any couple of pure unit quaternions that are orthogonal when they are regarded as vectors of $\mathbb{R}^{4}$. The quadruple $(1, v, w, v w)$ is then a direct orthonormal basis of $\mathbb{H} \cong \mathbb{R}^{4}$. For any hedgehog of $\mathbb{R}^{4}$ with support function $h \in C^{\infty}\left(\mathbb{S}^{3} ; \mathbb{R}\right)$ and, for any $u \in \mathbb{S}^{3}$, we have the following decompositions

$$
\begin{aligned}
x_{h}(u) & =h(u) u+\nabla h(u) \\
& =h(u) u+(\langle\nabla h(u), v u\rangle v u+\langle\nabla h(u), w u\rangle w u+\langle\nabla h(u), v w u\rangle v w u) \\
& =h(u) u+\partial_{v} h(v u) v u+\partial_{w} h(w u) w u+\partial_{v w} h(v w u) v w u \\
& =\left(h(u)+\partial_{v} h(v u) v+\partial_{w} h(w u) w+\partial_{v w} h(v w u) v w\right) u
\end{aligned}
$$

In particular, the hedgehog $x_{h}: \mathbb{S}^{3} \rightarrow \mathbb{H} \cong \mathbb{R}^{4}$ is the sum of parametrizations of 4 remarkable pedal surfaces: its own pedal surface and the pedal surfaces of its evolutes with respect to $v, w, v w$ (it being understood that, for all $u \in \mathbb{S}^{3}$, and, any pure unit quaternion $q$, we take the foot of the perpendicular from the origin to the support hyperplane with unit normal vector $J_{q}(u):=q u$ ).

\section{Evolutes and orthogonal projections}

For every $(u, v) \in \mathbb{S}^{3} \times \mathbb{S}^{2}$, let $\mathbb{S}_{u, v}^{1}$ be the oriented geodesic of $\mathbb{S}^{3}$ through $u$ in the direction of $J_{v}(u)$. This oriented circle of $\mathbb{S}^{3}$ can be regarded as the unit circle of the vector plane $\mathbb{C}(u, v):=\mathbb{R} u+\mathbb{R} J_{v}(u)$ oriented by $\left(u, J_{v}(u)\right)$. Restriction of support functions to $\mathbb{S}_{u, v}^{1}$ commutes with taking the evolutes in $\left(\mathbb{R}^{4}, J_{v}, \omega_{v}\right)$ :

Proposition 13 Let $h \in C^{\infty}\left(\mathbb{S}^{3} ; \mathbb{R}\right)$. For all $v \in \mathbb{S}^{2} \cong \mathbb{S}_{\mathbb{H}}^{1} \cap \operatorname{Im}(\mathbb{H})$,

$$
\left(\partial_{v} h\right)_{\mid \mathbb{S}_{u, v}^{1}}=\partial_{v}\left(h_{\mid \mathbb{S}_{u, v}^{1}}\right) .
$$

Proof. Define $u_{\theta}:=(\cos \theta) u+(\sin \theta) J_{v}(u)$ for all $\theta \in \mathbb{S}^{1}$. We have then

$$
\left(\partial_{v} h\right)\left(J_{v}\left(u_{\theta}\right)\right)=\left\langle\nabla h\left(u_{\theta}\right), J_{v}\left(u_{\theta}\right)\right\rangle=\frac{d}{d \theta}\left[h\left(u_{\theta}\right)\right]=\partial_{v}\left(h_{\mathbb{S}_{u, v}^{1}}\right)\left(J_{v}\left(u_{\theta}\right)\right) .
$$

\section{Higher order evolutes}

Of course, we can define inductively higher order evolutes. Let $\partial_{v}^{0} h=h$ and, for any positive integer $n$, define the $n$th evolute of $\mathcal{H}_{h}$ in $\left(\mathbb{R}^{4}, J_{v}, \omega_{v}\right)$ to be the hedgehog with support function $\partial_{v}^{n} h:=\partial_{v}\left(\partial_{v}^{n-1} h\right)$.

Proposition 14 Let $C^{\infty}\left(\mathbb{S}^{3} ; \mathbb{R}\right)$. For all $n \in \mathbb{N}^{*}, v \in \mathbb{S}^{2}$, and $u \in \mathbb{S}^{3}$,

$$
\left(\partial_{v}^{n} h\right)\left(J_{v}^{n}(u)\right)=\frac{d^{n}}{d \theta^{n}}\left[h\left(u_{\theta}\right)\right]_{\mid \theta=0},
$$

where $u_{\theta}:=(\cos \theta) u+(\sin \theta) J_{v}(u)$. 
Proof. By induction, we deduce from the previous proposition that

$$
\forall n \in \mathbb{N}^{*}, \quad\left(\partial_{v}^{n} h\right)_{\mathbb{S}_{u, v}^{1}}=\partial_{v}^{n}\left(h_{\mathbb{S}_{u, v}^{1}}\right),
$$

and the result follows immediately.

\subsection{Symplectic and mixed symplectic area}

Any pure unit quaternion $v$ determines a linear complex structure $J_{v}: \mathbb{R}^{4} \rightarrow \mathbb{R}^{4}$, to which it corresponds a Hopf flow induced on $\mathbb{S}^{3}=\mathbb{S}_{\mathbb{H}}^{1}$ by the vector field $X_{v}(u):=J_{v}(u)$. We denote by $\mathbb{S}^{2}$ the set $\mathbb{S}^{3} \cap \operatorname{Im}(\mathbb{H})$ of pure unit quaternions. For every $(u, v) \in \mathbb{S}^{3} \times \mathbb{S}^{2}$, let $\mathbb{S}_{u, v}^{1}$ be the oriented geodesic of $\mathbb{S}^{3}$ through $u$ in the direction of $J_{v}(u)$. This oriented Hopf circle of $\mathbb{S}^{3} \subset\left(\mathbb{R}^{4}, J_{v}\right)$ can be regarded as the unit circle of the vector plane $\mathbb{C}(u, v):=\mathbb{R} u+\mathbb{R} J_{v}(u)$ oriented by $\left(u, J_{v}(u)\right)$. Conversely, any oriented vector plane $\xi$ in $\mathbb{R}^{4}$ determines an oriented unit circle $\mathbb{S}_{\xi}^{1}=\mathbb{S}^{3} \cap \xi$ and a pure unit quaternion $v_{\xi}$ that is such that: $\forall u \in \mathbb{S}_{\xi}^{1}, T_{u} \mathbb{S}_{\xi}^{1}$ is oriented by the unit vector $J_{v_{\xi}}(u)$.

Now, consider the integral

$$
s_{\xi}(h):=\int_{x_{h}\left(\mathbb{S}_{\xi}^{1}\right)} \alpha_{v_{\xi}},
$$

where $\alpha_{v_{\xi}}$ is the 1-form given by $\left(\alpha_{v_{\xi}}\right)_{x}(d x)=\frac{1}{2} \omega_{v_{\xi}}(x, d x)$, which is such that $d \alpha_{v_{\xi}}=\omega_{v_{\xi}}$. This integral does not depend on the orientation of the plane $\xi$ (if we change the orientation of $\xi$, the orientation of the curve $x_{h}: \mathbb{S}_{\xi}^{1} \rightarrow \mathbb{R}^{4}$ changes as well and the 1 -form $\alpha_{v_{\xi}}$ is changed into its opposite). Therefore, $s_{\xi}(h)$ can be defined for any unoriented vector plane $\xi$ in $\mathbb{R}^{4}$. It will be called the symplectic area of $x_{h}\left(\mathbb{S}_{\xi}^{1}\right)$ relative to $\xi$.

\section{Expression of the symplectic area of $x_{h}\left(\mathbb{S}_{u, v}^{1}\right)$}

Let $s_{u, v}(h)$ be this symplectic area:

$$
s_{u, v}(h):=\int_{x_{h}\left(\mathbb{S}_{u, v}^{1}\right)} \alpha_{v},
$$

where $\alpha_{v}$ is the 1 -form given by $\left(\alpha_{v}\right)_{x}(d x):=\frac{1}{2} \omega_{v}(x, d x)=\frac{1}{2}\left\langle x,\left(-J_{v}\right)(d x)\right\rangle$.

Proposition 15 For all $h \in C^{\infty}\left(\mathbb{S}^{3} ; \mathbb{R}\right)$ and $(u, v) \in \mathbb{S}^{3} \times \mathbb{S}^{2}$,

$$
s_{u, v}(h)=\frac{1}{2} \int_{0}^{2 \pi}\left\langle x_{h}\left(u_{\theta}\right), R_{h}\left(u_{\theta}, v\right) u_{\theta}\right\rangle d \theta,
$$

where $u_{\theta}:=(\cos \theta) u+(\sin \theta) J_{v}(u)$ and $R_{h}\left(u_{\theta}, v\right):=-v\left(T_{u_{\theta}} x_{h}\right)\left(J_{v}\left(u_{\theta}\right)\right) \overline{u_{\theta}}$.

Proof. By definition 


$$
s_{u, v}(h):=\int_{x_{h}\left(\mathbb{S}_{u, v}^{1}\right)} \alpha_{v}=\frac{1}{2} \int_{0}^{2 \pi}\left\langle x_{h}\left(u_{\theta}\right),\left(-J_{v}\right)\left(\frac{d}{d \theta}\left[x_{h}\left(u_{\theta}\right)\right]\right)\right\rangle d \theta .
$$

Now

$$
\frac{d}{d \theta}\left[x_{h}\left(u_{\theta}\right)\right]=\left(T_{u_{\theta}} x_{h}\right)\left(J_{v}\left(u_{\theta}\right)\right)
$$

and hence

$$
\left(-J_{v}\right)\left(\frac{d}{d \theta}\left[x_{h}\left(u_{\theta}\right)\right]\right)=-v\left(T_{u_{\theta}} x_{h}\right)\left(J_{v}\left(u_{\theta}\right)\right)=R_{h}\left(u_{\theta}, v\right) u_{\theta} .
$$

Proposition 16 For all $h \in C^{\infty}\left(\mathbb{S}^{3} ; \mathbb{R}\right)$ and $(u, v) \in \mathbb{S}^{3} \times \mathbb{S}^{2}$,

$$
s_{u, v}(h)=a_{u, v}(h)+s_{u, v}^{\perp}(\nabla h)
$$

where $a_{u, v}(h)$ is the algebraic area of the hedgehog of $\mathbb{C}(u, v)=\mathbb{R} u+\mathbb{R} J_{v}(u)$ whose support function is the restriction of $h$ to $\mathbb{S}_{u, v}^{1}$, and where $s_{u, v}^{\perp}(\nabla h)$ is the symplectic area of $\nabla h\left(\mathbb{S}_{u, v}^{1}\right)$ in the Kähler vector space $\left(\mathbb{R}^{4}, J_{v}, \omega_{v}\right)$, that is,

$$
s_{u, v}^{\perp}(\nabla h):=\int_{\nabla h\left(\mathbb{S}_{u, v}^{1}\right)} \alpha_{v}=\frac{1}{2} \int_{\nabla h\left(\mathbb{S}_{u, v}^{1}\right)} \omega_{v}(x, d x) .
$$

Proof. It is just the fact that the symplectic area of a closed curve in the Kähler vector space $\left(\mathbb{R}^{4}, J_{v}, \omega_{v}\right)$ is the sum of the algebraic areas of its projections onto the planes $\mathbb{C}(u, v)$ and $\mathbb{C}(u, v)^{\perp}$. In the present case, we can retrieve the result as follows.

Let $\theta \in \mathbb{S}^{1}$. We have $x_{h}\left(u_{\theta}\right)=h\left(u_{\theta}\right) u_{\theta}+\nabla h\left(u_{\theta}\right)$, and

$$
\begin{aligned}
R_{h}\left(u_{\theta}, v\right) u_{\theta} & =\left(-J_{v}\right)\left(T_{u_{\theta}} x_{h}\left(J_{v}\left(u_{\theta}\right)\right)\right) \\
& =\left(-J_{v}\right)\left(h\left(u_{\theta}\right) J_{v}\left(u_{\theta}\right)+\nabla_{J_{v}\left(u_{\theta}\right)} \nabla h\left(u_{\theta}\right)\right) \\
& =h\left(u_{\theta}\right) u_{\theta}+\left(-J_{v}\right)\left(\nabla_{J_{v}\left(u_{\theta}\right)} \nabla h\left(u_{\theta}\right)\right),
\end{aligned}
$$

where $\nabla$ is the Levi-Civita connection on $\mathbb{S}^{3}$. In addition,

$$
\begin{aligned}
\left\langle u_{\theta},\left(-J_{v}\right)\left(\nabla_{J_{v}\left(u_{\theta}\right)} \nabla h\left(u_{\theta}\right)\right)\right\rangle & =\left\langle J_{v}\left(u_{\theta}\right), \nabla_{J_{v}\left(u_{\theta}\right)} \nabla h\left(u_{\theta}\right)\right\rangle \\
& =\frac{d}{d \theta}\left[\left\langle J_{v}\left(u_{\theta}\right), \nabla h\left(u_{\theta}\right)\right\rangle\right]=\frac{d^{2}}{d \theta^{2}}\left[h\left(u_{\theta}\right)\right],
\end{aligned}
$$

and

$$
\left\langle\nabla h\left(u_{\theta}\right),\left(-J_{v}\right)\left(\nabla_{J_{v}\left(u_{\theta}\right)} \nabla h\left(u_{\theta}\right)\right)\right\rangle=\omega_{v}\left(\nabla h\left(u_{\theta}\right), \frac{d}{d \theta}\left[\nabla h\left(u_{\theta}\right)\right]\right)
$$


since $\frac{d}{d \theta}\left[\nabla h\left(u_{\theta}\right)\right]=\nabla_{J_{v}\left(u_{\theta}\right)} \nabla h\left(u_{\theta}\right)-\left\langle\nabla h\left(u_{\theta}\right), J_{v}\left(u_{\theta}\right)\right\rangle u_{\theta}$. Hence

$$
\left\langle x_{h}\left(u_{\theta}\right),\left(-J_{v}\right)\left(\frac{d}{d \theta}\left[x_{h}\left(u_{\theta}\right)\right]\right)\right\rangle=h\left(u_{\theta}\right)\left(h\left(u_{\theta}\right)+\frac{d^{2}}{d \theta^{2}}\left[h\left(u_{\theta}\right)\right]\right)+\omega_{v}\left(\nabla h\left(u_{\theta}\right), \frac{d}{d \theta}\left[\nabla h\left(u_{\theta}\right)\right]\right)
$$

The result is then an immediate consequence of the previous proposition.

\section{Mixed symplectic area}

Proposition 17 (Symmetry) For all $(f, g) \in C^{\infty}\left(\mathbb{S}^{3} ; \mathbb{R}\right)^{2}$, and $(u, v) \in \mathbb{S}^{3} \times \mathbb{S}^{2}$,

$$
\int_{0}^{2 \pi}\left\langle x_{f}\left(u_{\theta}\right), R_{g}\left(u_{\theta}, v\right) u_{\theta}\right\rangle d \theta=\int_{0}^{2 \pi}\left\langle x_{g}\left(u_{\theta}\right), R_{f}\left(u_{\theta}, v\right) u_{\theta}\right\rangle d \theta .
$$

Proof. For all $\theta \in \mathbb{S}^{1}$,

$$
\frac{d}{d \theta}\left[\omega_{v}\left(x_{f}\left(u_{\theta}\right), x_{g}\left(u_{\theta}\right)\right)\right]=\omega_{v}\left(T_{u_{\theta}} x_{f}\left(J_{v}\left(u_{\theta}\right)\right), x_{g}\left(u_{\theta}\right)\right)+\omega_{v}\left(x_{f}\left(u_{\theta}\right), T_{u_{\theta}} x_{g}\left(J_{v}\left(u_{\theta}\right)\right)\right) .
$$

By integration, we deduce that

$$
\int_{0}^{2 \pi} \omega_{v}\left(x_{f}\left(u_{\theta}\right), T_{u_{\theta}} x_{g}\left(J_{v}\left(u_{\theta}\right)\right)\right) d \theta=\int_{0}^{2 \pi} \omega_{v}\left(x_{g}\left(u_{\theta}\right), T_{u_{\theta}} x_{f}\left(J_{v}\left(u_{\theta}\right)\right)\right) d \theta
$$

which is the desired equality since

$$
\begin{aligned}
\omega_{v}\left(x_{h}\left(u_{\theta}\right), T_{u_{\theta}} x_{h}\left(J_{v}\left(u_{\theta}\right)\right)\right) & =\left\langle J_{v} x_{h}\left(u_{\theta}\right), T_{u_{\theta}} x_{h}\left(J_{v}\left(u_{\theta}\right)\right)\right\rangle \\
& =\left\langle x_{h}\left(u_{\theta}\right),-J_{v}\left[T_{u_{\theta}} x_{h}\left(J_{v}\left(u_{\theta}\right)\right)\right]\right\rangle \\
& =\left\langle x_{h}\left(u_{\theta}\right), R_{h}\left(u_{\theta}, v\right) u_{\theta}\right\rangle
\end{aligned}
$$

for $h \in\{f, g\}$.

Definition 18 Let $(f, g) \in C^{\infty}\left(\mathbb{S}^{3} ; \mathbb{R}\right)^{2}$ and $(u, v) \in \mathbb{S}^{3} \times \mathbb{S}^{2}$. We call $s_{u, v}(f, g):=\frac{1}{2}\left(s_{u, v}(f+g)-s_{u, v}(f)-s_{u, v}(g)\right)=\frac{1}{2} \int_{0}^{2 \pi}\left\langle x_{f}\left(u_{\theta}\right), R_{g}\left(u_{\theta}, v\right) u_{\theta}\right\rangle d \theta$ the mixed symplectic area of $x_{f}\left(\mathbb{S}_{u, v}^{1}\right)$ and $x_{g}\left(\mathbb{S}_{u, v}^{1}\right)$.

A straightforward computation shows that

$$
s_{u, v}(f, g)=a_{u, v}(f, g)+s_{u, v}^{\perp}(\nabla f, \nabla g),
$$

where $a_{u, v}(f, g)$ is the mixed symplectic area of the hedgehogs of $\mathbb{C}(u, v)$ with support functions $f_{\mid \mathbb{S}_{u, v}^{1}}$ and $g_{\mid \mathbb{S}_{u, v}^{1}}$, and where $s_{u, v}^{\perp}(\nabla f, \nabla g)$ is the mixed symplectic area of $\nabla f\left(\mathbb{S}_{u, v}^{1}\right)$ and $\nabla g\left(\mathbb{S}_{u, v}^{1}\right)$ in $\left(\mathbb{R}^{4}, J_{v}, \omega_{v}\right)$, that is, 


$$
\begin{aligned}
s_{u, v}^{\perp}(\nabla f, \nabla g) & :=\frac{1}{2}\left(s_{u, v}^{\perp}(\nabla(f+g))-s_{u, v}^{\perp}(\nabla f)-s_{u, v}^{\perp}(\nabla g)\right) \\
& =\frac{1}{2} \int_{0}^{2 \pi} \omega_{v}\left(\nabla f\left(u_{\theta}\right), \frac{d}{d \theta}\left[\nabla g\left(u_{\theta}\right)\right]\right) d \theta \\
& =\frac{1}{2} \int_{0}^{2 \pi} \omega_{v}\left(\nabla g\left(u_{\theta}\right), \frac{d}{d \theta}\left[\nabla f\left(u_{\theta}\right)\right]\right) d \theta .
\end{aligned}
$$

\section{Symplectic area of $\mathcal{H}_{h}$}

Definition 19 Let $h \in C^{\infty}\left(\mathbb{S}^{3} ; \mathbb{R}\right)$. We define the symplectic area of $\mathcal{H}_{h}$ to be

$$
s(h):=\frac{v_{4}}{v_{2}} \int_{G_{4,2}} s_{\xi}(h) d \omega_{4,2}(\xi),
$$

where $v_{n+1}$ is the volume of the unit ball in $\mathbb{R}^{n+1}, G_{4,2}$ the Grassman manifold of 2-dimensional subspaces of $\mathbb{R}^{4}$ and $\omega_{4,2}$ the normalized Haar measure on $G_{4,2}$ : $\omega_{4,2}\left(G_{4,2}\right)=1$.

Recall that the mixed volume $V:\left(\mathcal{K}^{4}\right)^{4} \rightarrow \mathbb{R}$ extends to a symmetric 4 -linear form on the vector space $\mathcal{H}^{4}$ of hedgehogs of $\mathbb{R}^{4}$. Besides, the algebraic area of order 2 of a hedgehog $\mathcal{H}_{h}$ of $\mathbb{R}^{4}$, denoted by $V_{2}(h)$, is defined to be the mixed volume $V(h, h, 1,1)$.

Proposition 20 For any $h \in C^{\infty}\left(\mathbb{S}^{3} ; \mathbb{R}\right)$, the symplectic area of $\mathcal{H}_{h}$ is equal to its algebraic area of order 2 , that is, $s(h):=V_{2}(h)$.

Proof. From Kubota's formula

$$
V_{2}(K)=\frac{v_{4}}{v_{2}} \int_{G_{4,2}} V\left(p_{\xi}(K)\right) d \omega_{4,2}(\xi),
$$

for all convex body $K$ in $\mathbb{R}^{4}$, where $p_{\xi}(K)$ is the orthogonal projection of $K$ on $\xi \in G_{4,2}, V\left(p_{\xi}(K)\right)$ its area and $V_{2}(K)$ the mixed volume $V(K, K, B, B)$, $B$ denoting the unit ball in $\mathbb{R}^{4}$ (see [16, Section 5.3]). This formula can be extended to hedgehogs by multilinearity, so that:

$$
v_{2}(h)=\frac{v_{4}}{v_{2}} \int_{G_{4,2}} a\left(h_{\mid \mathbb{S}_{\xi}^{1}}\right) d \omega_{4,2}(\xi),
$$

for all $h \in C^{\infty}\left(\mathbb{S}^{3} ; \mathbb{R}\right)$. Note that the algebraic area of $\mathcal{H}_{h_{\mid \mathbb{S}_{\xi}^{1}}}$ does not depend on a choice of orientation for $\xi$. Now, we have proved above that

$$
s_{u, v}(h)=a\left(h_{\mid S_{u, v}^{1}}\right)+s_{u, v}^{\perp}(\nabla h)
$$


for all $(u, v) \in \mathbb{S}^{3} \times \mathbb{S}^{2}$. So, it suffices to prove that for all $u \in \mathbb{S}^{3}$,

$$
\int_{\mathbb{S}^{2}} \omega_{v}\left(\nabla h(u), \nabla_{J_{v}(u)} \nabla h(u)\right) d \sigma(v)=0,
$$

where $\sigma$ is the spherical Lebesgue measure.

Now, let $\left(v_{1}, v_{2}, v_{3}\right) \in \mathbb{S}^{2}=\mathbb{S}_{\mathbb{H}}^{1} \cap \operatorname{Im}(\mathbb{H})$ be such that $\left(J_{v_{1}}(u), J_{v_{2}}(u), J_{v_{3}}(u)\right)$ is an orthonormal basis of $T_{u} \mathbb{S}^{3}$ formed by eigenvectors of the Riemannian Hessian $\left(\nabla^{2} h\right)_{u}$, corresponding to eigenvalues $\left(\lambda_{1}, \lambda_{2}, \lambda_{3}\right)$. The product of two imaginary quaternions $q_{1}, q_{2} \in \operatorname{Im}(\mathbb{H})=\mathbb{R}^{3}$ is given by $q_{1} q_{2}=-\left\langle q_{1}, q_{2}\right\rangle+q_{1} \times q_{2}$, where $\langle.,$.$\rangle is the Euclidean inner product and \times$ the usual vector product on $\mathbb{R}^{3}$. Since the orthonormal basis $\left(v_{1}, v_{2}, v_{3}\right)$ is formed by imaginary quaternions, we thus have: $v_{i} v_{j}+v_{j} v_{i}=0$ for all $(i, j) \in[1,3]^{2}$ such that $i \neq j$. A straightforward calculation then gives, for any $v=\sum_{i=1}^{3} x_{i} v_{i} \in \mathbb{S}^{2}$,

$$
\begin{aligned}
\left(-J_{v}\right)\left(\nabla_{J_{v}(u)} \nabla h(u)\right) & =\left(-J_{v}\right)\left(\sum_{j=1}^{3} x_{j} \lambda_{j} J_{v_{j}}(u)\right)=\sum_{i, j=1}^{3} x_{i} x_{j} \lambda_{i} \lambda_{j} v_{i} v_{j} u \\
& =\sum_{1 \leq i<j \leq 3} x_{i} x_{j}\left(\lambda_{i}-\lambda_{j}\right) v_{i} v_{j} u
\end{aligned}
$$

and hence

$$
\begin{aligned}
\int_{\mathbb{S}^{2}} \omega_{v}\left(\nabla h(u), \nabla_{J_{v}(u)} \nabla h(u)\right) d \sigma(v) & =\int_{\mathbb{S}^{2}}\left(-J_{v}\right)\left(\nabla_{J_{v}(u)} \nabla h(u)\right) d \sigma(v) \\
& =\sum_{1 \leq i<j \leq 3}(\underbrace{\int_{\mathbb{S}^{2}} x_{i} x_{j} d \sigma}_{=0})\left(\lambda_{i}-\lambda_{j}\right) v_{i} v_{j} u . \\
& =0
\end{aligned}
$$

which achieves the proof.

\subsection{Quaternionic curvature function}

Let $K$ be a convex body with class $C_{+}^{\infty}$ in $(n+1)$-Euclidean vector space $\mathbb{R}^{n+1}$. One says that $K$ has the $\left(C^{\infty}\right.$-smooth) curvature function $R_{K}: \mathbb{S}^{n} \rightarrow \mathbb{R}$ if its surface area measure $S_{n}(K,$.$) has R_{K}$ as density with respect to spherical area measure $\sigma$ or, equivalently, if

$$
\begin{aligned}
V(L, K, \ldots, K) & =\frac{1}{n+1} \int_{\mathbb{S}^{n}} h_{L}(u) R_{K}(u) d \sigma(u) \\
& =\frac{1}{n+1} \int_{\mathbb{S}^{n}}\left\langle x_{h_{L}}(u), R_{K}(u) u\right\rangle d \sigma(u),
\end{aligned}
$$


for all convex body $L$ with support function $h_{L}: \mathbb{S}^{n} \rightarrow \mathbb{R}$ (see e.g. [16, p. $545])$. The notion of curvature function naturally extends to $C^{2}$-hedgehogs of $\mathbb{R}^{n+1}[10]$. The aim of this subsection is to use the notion of the mixed symplectic area of $x_{g}\left(\mathbb{S}_{u, v}^{1}\right)$ and $x_{h}\left(\mathbb{S}_{u, v}^{1}\right)$ to introduce the notion of the (quaternionic) curvature function of $x_{h}\left(\mathbb{S}_{u, v}^{1}\right)$. As already mentioned, the reason why the map $\theta \longmapsto R_{h}\left(e^{i \theta}\right)$ is real for $h \in C^{\infty}\left(\mathbb{S}^{1} ; \mathbb{R}\right)$, whereas $u \mapsto R_{h}(u, v)$ is quaternionic for $h \in C^{\infty}\left(\mathbb{S}^{3} ; \mathbb{R}\right)$, is because the product of two purely imaginary complex numbers is a real number, whereas the product of two purely imaginary quaternions can have both nontrivial real and imaginary parts.

Proposition 21 Let $h \in C^{\infty}\left(\mathbb{S}^{3} ; \mathbb{R}\right)$, and $(u, v) \in \mathbb{S}^{3} \times \mathbb{S}^{2}$. There exists one and only one $C^{\infty}$-smooth quaternionic function $R(., v): \mathbb{S}_{u, v}^{1} \rightarrow \mathbb{H}$ that is of the form

$$
u_{\theta}:=(\cos \theta) u+(\sin \theta) v u \longmapsto R\left(u_{\theta}, v\right)=-v T_{u_{\theta}}(v),
$$

where $T_{u_{\theta}}(v)$ denotes a pure quaternion, and such that:

$$
\forall g \in C^{\infty}\left(\mathbb{S}^{3} ; \mathbb{R}\right), \quad s_{u, v}(g, h):=\frac{1}{2} \int_{0}^{2 \pi}\left\langle x_{g}\left(u_{\theta}\right), R\left(u_{\theta}, v\right) u_{\theta}\right\rangle d \theta,
$$

where $u_{\theta}:=(\cos \theta) u+(\sin \theta) v u$. Namely, the quaternionic function given by: $R_{h}\left(u_{\theta}, v\right):=-v\left(T_{u_{\theta}} x_{h}\right)\left(v u_{\theta}\right) \overline{u_{\theta}}$ for all $\theta \in \mathbb{S}^{1}$.

Proof. For all $\theta \in \mathbb{S}^{1}, T_{u_{\theta}} \mathbb{S}^{3}=\operatorname{Im}(\mathbb{H}) u_{\theta}$ and hence $\left(T_{u_{\theta}} x_{h}\right)\left(v u_{\theta}\right) \overline{u_{\theta}} \in \operatorname{Im}(\mathbb{H})$. Thus, $R_{h}(., v): u_{\theta} \longmapsto R_{h}\left(u_{\theta}, v\right)=-v\left(T_{u_{\theta}} x_{h}\right)\left(v u_{\theta}\right) \overline{u_{\theta}}$ is of the required form since

$$
\forall g \in C^{\infty}\left(\mathbb{S}^{3} ; \mathbb{R}\right), \quad s_{u, v}(g, h):=\frac{1}{2} \int_{0}^{2 \pi}\left\langle x_{g}\left(u_{\theta}\right), R_{h}\left(u_{\theta}, v\right) u_{\theta}\right\rangle d \theta
$$

Conversely, let $R(., v)$ be any function satisfying the required conditions. Note that the map $u_{\theta} \mapsto R\left(u_{\theta}, v\right) u_{\theta}$ has then the form $u_{\theta} \mapsto \rho\left(u_{\theta}\right) u_{\theta}+\rho^{\perp}\left(u_{\theta}\right)$, where $\rho \in C^{\infty}\left(\mathbb{S}_{u, v}^{1} ; \mathbb{R}\right)$ and $\rho^{\perp} \in C^{\infty}\left(\mathbb{S}_{u, v}^{1} ; \mathbb{C}(u, v)^{\perp}\right)$. Indeed, we have

$$
\left\langle R\left(u_{\theta}, v\right) u_{\theta}, J_{v}\left(u_{\theta}\right)\right\rangle=\left\langle J_{v}\left(-T_{u_{\theta}}(v) u_{\theta}\right), J_{v}\left(u_{\theta}\right)\right\rangle=\left\langle-T_{u_{\theta}}(v) u_{\theta}, u_{\theta}\right\rangle=0
$$

for all $\theta \in \mathbb{S}^{1}$, since $-T_{u_{\theta}}(v) u_{\theta} \in \operatorname{Im}(\mathbb{H}) u_{\theta}=T_{u_{\theta}} \mathbb{S}^{3}$. Besides, in the case where $R(., v)=R_{h}(., v)$, we have $R\left(u_{\theta}, v\right) u_{\theta}=R_{h}\left(u_{\theta}, v u_{\theta}\right) u_{\theta}-v \pi_{u, v}^{\perp}\left[\nabla_{v u_{\theta}} \nabla h\left(u_{\theta}\right)\right]$, where $R_{h}\left(u_{\theta}, v u_{\theta}\right)$ is the radius of curvature of $x_{h_{\mid \mathbb{S}_{u, v}^{1}}}: \mathbb{S}_{u, v}^{1} \rightarrow \mathbb{C}(u, v)$ at $x_{h_{\mid \mathbb{S}_{u, v}^{1}}}\left(u_{\theta}\right)$ (or, equivalently, the tangential radius of curvature of $\mathcal{H}_{h}$ at $x_{h}\left(u_{\theta}\right)$ in the direction $v u_{\theta}$, which is given by: $R_{h}\left(u_{\theta}, v u_{\theta}\right):=\left\langle T_{u_{\theta}} x_{h}\left(v u_{\theta}\right), v u_{\theta}\right\rangle=$ $h\left(u_{\theta}\right)+\left(\nabla^{2} h\right)_{u_{\theta}}\left(v u_{\theta}, v u_{\theta}\right)$; see e.g. [10]), and $\pi_{u, v}^{\perp}$ the orthogonal projection onto the subspace of $\mathbb{R}^{4}$ that is orthogonal to $\mathbb{C}(u, v)$. Indeed,

$$
\begin{aligned}
R_{h}\left(u_{\theta}, v\right) u_{\theta} & =-v\left(T_{u_{\theta}} x_{h}\right)\left(v u_{\theta}\right) \\
& =-v\left(h\left(u_{\theta}\right) v u_{\theta}+\nabla_{v u_{\theta}} \nabla h\left(u_{\theta}\right)\right) \\
& =-v\left(\left(h\left(u_{\theta}\right)+\left(\nabla^{2} h\right)_{u_{\theta}}\left(v u_{\theta}, v u_{\theta}\right)\right) v u_{\theta}+\pi_{u, v}^{\perp}\left[\nabla_{v u_{\theta}} \nabla h\left(u_{\theta}\right)\right]\right)
\end{aligned}
$$


We already know that $\mathbb{S}_{u, v}^{1} \rightarrow \mathbb{R}, u_{\theta} \mapsto R_{h}\left(u_{\theta}, v u_{\theta}\right)$ is the unique $C^{\infty}$-smooth function $R: \mathbb{S}_{u, v}^{1} \rightarrow \mathbb{R}$ that satisfies:

$$
\forall g \in C^{\infty}\left(\mathbb{S}_{u, v}^{1} ; \mathbb{R}\right), \quad a_{u, v}(g, h):=\frac{1}{2} \int_{0}^{2 \pi} g\left(u_{\theta}\right) R\left(u_{\theta}\right) d \theta .
$$

Now, any $g \in C^{\infty}\left(\mathbb{S}_{u, v}^{1} ; \mathbb{R}\right)$ can be extended into a function $g_{S} \in C^{\infty}\left(\mathbb{S}^{3} ; \mathbb{R}\right)$ that is such that $\pi_{u, v}^{\perp}\left[\left(\nabla g_{S}\right)_{\mid \mathbb{S}_{u, v}^{1}}\right]=0$ : it suffices, for instance, to define $g_{S}$ by

$$
\forall q \in \mathbb{S}^{3}, \quad g_{S}(q):=\left\{\begin{array}{l}
0 \quad \text { if } \quad\|p\|=0 \\
\digamma(\|p\|) g\left(\frac{p}{\|p\|}\right) \quad \text { if } \quad\|p\| \neq 0,
\end{array}\right.
$$

where $p$ is the orthogonal projection of $q$ onto $\mathbb{C}(u, v)$ and,

$$
\digamma(t):=\frac{\int_{0}^{t} \varphi(\tau) \varphi(1-\tau) d \tau}{\int_{0}^{1} \varphi(\tau) \varphi(1-\tau) d \tau},
$$

where $\varphi$ is the function defined on $\mathbb{R}$ by

$$
\varphi(t):=\left\{\begin{array}{l}
0 \quad \text { if } \quad \tau \leq 0 \\
e^{-\frac{1}{t^{2}}} \quad \text { if } \quad \tau>0 .
\end{array} .\right.
$$

$\left(F: \mathbb{R} \rightarrow \mathbb{R}\right.$ is $C^{\infty}$-smooth, and such that $F(0)=0, F(1)=1$, and: $\forall n \in \mathbb{N}^{*}$, $\left.F^{(n)}(0)=F^{(n)}(1)=0\right)$. For any $g \in C^{\infty}\left(\mathbb{S}_{u, v}^{1} ; \mathbb{R}\right)$, such an extension $g_{S}$ is such that

$$
s_{u, v}\left(g_{S}, h\right)=a_{u, v}(g, h)=\frac{1}{2} \int_{0}^{2 \pi} g\left(u_{\theta}\right) \rho\left(u_{\theta}\right) d \theta,
$$

since $s_{u, v}^{\perp}\left(\nabla g_{S}, \nabla h\right)=0$. Therefore $\rho\left(u_{\theta}\right)=R_{h}\left(u_{\theta}, v u_{\theta}\right)$ for all $\theta \in \mathbb{S}^{1}$.

Now, it remains to prove that $\rho^{\perp}\left(u_{\theta}\right)=-v \pi_{u, v}^{\perp}\left[\nabla_{v u_{\theta}} \nabla h\left(u_{\theta}\right)\right]$ for all $\theta \in \mathbb{S}^{1}$. Since $\rho\left(u_{\theta}\right)=R_{h}\left(u_{\theta}, v u_{\theta}\right)$ for all $\theta \in \mathbb{S}^{1}$, the integral condition can be rewritten as follows:

$$
\forall g \in C^{\infty}\left(\mathbb{S}^{3} ; \mathbb{R}\right), \quad s_{u, v}^{\perp}(\nabla g, \nabla h):=\frac{1}{2} \int_{0}^{2 \pi}\left\langle\nabla g\left(u_{\theta}\right)^{\perp}, \rho^{\perp}\left(u_{\theta}\right)\right\rangle d \theta,
$$

where $\nabla g\left(u_{\theta}\right)^{\perp}:=\pi_{u, v}^{\perp}\left[\nabla g\left(u_{\theta}\right)\right]$, that is,

$$
\forall g \in C^{\infty}\left(\mathbb{S}^{3} ; \mathbb{R}\right), \quad \int_{0}^{2 \pi}\left\langle\nabla g\left(u_{\theta}\right)^{\perp}, \rho^{\perp}\left(u_{\theta}\right)+v \pi_{u, v}^{\perp}\left[\nabla_{v u_{\theta}} \nabla h\left(u_{\theta}\right)\right]\right\rangle d \theta=0 .
$$

Note that $\nabla g\left(u_{\theta}\right)^{\perp}$ has the form

$$
\nabla g\left(u_{\theta}\right)^{\perp}=\left(\left\langle\nabla g\left(u_{\theta}\right), w u_{\theta}\right\rangle+\left\langle\nabla g\left(u_{\theta}\right), v w u_{\theta}\right\rangle v\right) w u_{\theta},
$$


where $w$ is a pure unit quaternion that is $\langle.,$.$\rangle -orthogonal to v$, so that $(v, w, v w)$ is an orthonormal basis of $\operatorname{Im}(\mathbb{H})$. Moreover, $\rho^{\perp}\left(u_{\theta}\right)+v \pi_{u, v}^{\perp}\left[\nabla_{v u_{\theta}} \nabla h\left(u_{\theta}\right)\right]$ has the form $\left(\lambda\left(u_{\theta}\right)+\mu\left(u_{\theta}\right) v\right) w u_{\theta}$, where $\lambda$ and $\mu$ are real, since it belongs to $\mathbb{R} w u_{\theta}+\mathbb{R} v w u_{\theta}=\mathbb{C}(u, v)^{\perp}$. Thus, the integral condition is that the function $\mathbb{S}_{u, v}^{1} \rightarrow \mathbb{C}(u, v)^{\perp}, u_{\theta} \longmapsto \rho^{\perp}\left(u_{\theta}\right)+v \pi_{u, v}^{\perp}\left[\nabla_{v u_{\theta}} \nabla h\left(u_{\theta}\right)\right]$ is $L^{2}$-orthogonal to all the functions $\mathbb{S}_{u, v}^{1} \rightarrow \mathbb{C}(u, v)^{\perp}, u_{\theta} \longmapsto \nabla g\left(u_{\theta}\right)^{\perp}$ where $g \in C^{\infty}\left(\mathbb{S}^{3} ; \mathbb{R}\right)$. Now, for any two real $C^{\infty}$-functions $a, b$ on $\mathbb{S}_{u, v}^{1}$, let us define $g: \mathbb{S}^{3} \rightarrow \mathbb{H}$ by:

$$
g(q(\theta, \beta, \gamma)):=\left[a\left(u_{\theta}\right)\left\langle q(\theta, \beta, \gamma), w u_{\theta}\right\rangle+b\left(u_{\theta}\right)\left\langle q(\theta, \beta, \gamma), v w u_{\theta}\right\rangle\right] F(\cos \beta),
$$

where

$$
\begin{aligned}
q(\theta, \beta, \gamma)= & (\cos \beta) u_{\theta}+(\sin \beta)\left((\cos \gamma) w u_{\theta}+(\sin \gamma) v w u_{\theta}\right) \in \mathbb{S}^{3} \\
= & (\cos \beta)(\cos \theta) u+(\cos \beta)(\sin \theta) v u+ \\
& \quad(\cos (\gamma-\theta)(\sin \beta)) w u+(\sin (\gamma-\theta))(\sin \beta) v w u .
\end{aligned}
$$

We then obtain

$$
\begin{aligned}
\frac{\partial}{\partial \beta}[g(q(\theta, \beta, \gamma))]_{\mid \beta=0} & =\left\langle\nabla g(q(\theta, 0, \gamma)), \frac{\partial q}{\partial \beta}(\theta, 0, \gamma)\right\rangle \\
& =\left\langle\nabla g\left(u_{\theta}\right),(\cos \gamma) w u_{\theta}+(\sin \gamma) v w u_{\theta}\right\rangle \\
& =a\left(u_{\theta}\right) \cos \gamma+b\left(u_{\theta}\right) \sin \gamma
\end{aligned}
$$

and thus, for $\gamma=0$ and $\gamma=\pi / 2$, we have respectively $a\left(u_{\theta}\right)=\left\langle\nabla g\left(u_{\theta}\right), w u_{\theta}\right\rangle$ and $b\left(u_{\theta}\right)=\left\langle\nabla g\left(u_{\theta}\right), v w u_{\theta}\right\rangle$. In other words, all the functions of the form $\mathbb{S}_{u, v}^{1} \rightarrow \mathbb{C}(u, v)^{\perp}, u_{\theta} \longmapsto\left(a\left(u_{\theta}\right)+b\left(u_{\theta}\right) v\right) w u_{\theta}$ can be written in the form $\mathbb{S}_{u, v}^{1} \rightarrow \mathbb{C}(u, v)^{\perp}, u_{\theta} \longmapsto \nabla g\left(u_{\theta}\right)^{\perp}$ where $g \in C^{\infty}\left(\mathbb{S}^{3} ; \mathbb{R}\right)$.

Therefore, $\rho^{\perp}\left(u_{\theta}\right)=-v \pi_{u, v}^{\perp}\left[\nabla_{v u_{\theta}} \nabla h\left(u_{\theta}\right)\right]$ for all $\theta \in \mathbb{S}^{1}$.

Definition 22 For every $h \in C^{\infty}\left(\mathbb{S}^{3} ; \mathbb{R}\right)$, we say that $R_{h}(., v): \mathbb{S}_{u, v}^{1} \rightarrow \mathbb{H}$, $u_{\theta} \longmapsto-v\left(T_{u_{\theta}} x_{h}\right)\left(v u_{\theta}\right) \overline{u_{\theta}}$ is the quaternionic curvature function of $x_{h}\left(\mathbb{S}_{u, v}^{1}\right)$.

\subsection{Convolution of hedgehogs}

Differences of (arbitrary) convex bodies of $\mathbb{R}^{2}$ do not only constitute a real vector space $\left(\mathcal{H}^{2},+,.\right)$ but also a commutative and associative $\mathbb{R}$-algebra. Indeed, as noticed by H. Görtler in [5] and [6], we can define the convolution product of two plane hedgehogs $\mathcal{H}_{f}$ and $\mathcal{H}_{g}$ in $\mathbb{R}^{2}$ as the plane hedgehog whose support function is given by

$$
(f * g)(\theta)=\frac{1}{2 \pi} \int_{0}^{2 \pi} f(\theta-\alpha) g(\alpha) d \alpha,
$$

for all $\theta \in \mathbb{S}^{1}$; and we can check at once that $\left(\mathcal{H}^{2},+, ., *\right)$ is then a commutative and associative algebra. H. Görtler also noticed that the convolution product of two plane convex bodies is still a plane convex body. The interest of convolution of hedgehogs is that properties of one factor are often transmitted to the product. 
Of course, we think immediately of regularity properties but we also mentioned the following properties in [12]: to be centered (centrally symmetric with center at the origin), to be projective (i.e., to have an antisymmetric support function), to be of constant width.

A natural way of defining a (non-abelian) convolution product on the vector space $\mathcal{H}^{n+1}$ of arbitrary hedgehogs of $\mathbb{R}^{n+1}$ is to proceed as follows: 1 . First, we identify $\mathbb{S}^{n}$ with the homogeneous space $G / H$, where $G$ is the group $S O(n+1)$ of rotations of $\mathbb{R}^{n+1}$ and $H$ the stabilizer subgroup of $G$ with respect to the north pole of $\mathbb{S}^{n}$, say $\nu$ (that is, the subgroup $H$ of $G$ formed by the rotations $r \in G$ that leave $\nu$ fixed); any support function $h: \mathbb{S}^{n} \rightarrow \mathbb{R}$ can thus be regarded as a function $h: G \rightarrow \mathbb{R}$ such that $h(r s)=h(r)$ for all $(r, s) \in G \times H ; 2$. Next, given any two arbitrary hedgehogs $\mathcal{H}_{f}$ and $\mathcal{H}_{g}$ of $\mathbb{R}^{n+1}$, we can define their convolution product $\mathcal{H}_{f} * \mathcal{H}_{g}$ as the hedgehog $\mathcal{H}_{f * g}$ with support function

$$
(f * g)(r)=\int_{G} f\left(r t^{-1}\right) g(t) d m_{G}(t) \quad \text { for all } r \in G,
$$

where $m_{G}$ is the normalized Haar measure on $G$. This construction of $\mathcal{H}_{f} * \mathcal{H}_{g}$ is essentially due to E. Grindberg and G. Zhang [7]. As expected, this convolution product behaves well with respect to expansions in series of spherical harmonics, and properties of one factor are often transmitted to the product (for instance, to be centred, projective, convex, of constant width, or a zonoid).

But of course, in the case of hedgehogs of $\mathbb{R}^{4}$ it is simpler to make use of quaternions and thus to define the convolution product $\mathcal{H}_{f} * \mathcal{H}_{g}$ of $\mathcal{H}_{f}$ and $\mathcal{H}_{g}$ in $\mathbb{R}^{4}$ to be the hedgehog $\mathcal{H}_{f * g}$ with support function

$$
(f * g)(u)=\int_{\mathbb{S}^{3}} f(v \bar{u}) g(v) d \sigma(v) \quad \text { for all } u \in \mathbb{S}_{\mathbb{H}}^{1} \cong \mathbb{S}^{3},
$$

where $\sigma$ is the spherical Lebesgue measure on $\mathbb{S}^{3}$.

\subsection{Evolutes of hedgehogs hypersurfaces in $\mathbb{H}^{n} \cong \mathbb{R}^{4 n}$}

We identify $\mathbb{R}^{4 n}$ with the hyperkähler vector space $\left(\mathbb{H}^{n},\langle.,\rangle, I, J, K.\right)$, where $\langle.,$.$\rangle is the standard Euclidean metric on \mathbb{R}^{4 n} \cong \mathbb{H}^{n},(n \geq 1)$, and, the triple of complex structures $(I, J, K)$ on $\mathbb{H}^{n}$ is given by left multiplication by $i, j, k$ respectively. On this hyperkähler vector space, we have a whole $\mathbb{S}^{2}$ family of linear Kähler structures given by:

$$
I_{a}:=a_{1} I+a_{2} J+a_{3} K \quad \text { and } \quad \omega_{a}(X, Y)=\left\langle I_{a}(X), Y\right\rangle,
$$

for all $a=\left(a_{1}, a_{2}, a_{3}\right) \in \mathbb{S}^{2} \subset \mathbb{R}^{3}$ and, $(X, Y) \in\left(T_{q} \mathbb{H}^{n}\right)^{2}$. Most of the results we saw for evolutes of hedgehogs in $\mathbb{R}^{4} \cong \mathbb{H}$ can be extended to $\left(\mathbb{H}^{n},\langle.,\rangle, I, J, K.\right)$ with a few adaptations. In particular, for all $h \in C^{\infty}\left(\mathbb{S}^{4 n-1} ; \mathbb{R}\right)$, the evolute of the hedgehog $\mathcal{H}_{h}$ in the Kähler vector space $\left(\mathbb{R}^{4}, I_{a}, \omega_{a}\right)$ is defined to be the envelope of the family of normal hyperplanes $\left(N_{h}^{a}(u)\right)_{u \in \mathbb{S}^{4 n-1}}$ with equation

$$
\left\langle x-x_{h}(u), I_{a}(u)\right\rangle=0 .
$$


Proposition 23 Let $h \in C^{\infty}\left(\mathbb{S}^{4 n-1} ; \mathbb{R}\right)$. The evolute of $\mathcal{H}_{h}$ in $\left(\mathbb{H}^{n}, I_{a}, \omega_{a}\right)$ is the hedgehog $\mathcal{H}_{\partial_{a} h}$ with support function

$$
\partial_{a} h: \mathbb{S}^{4 n-1} \rightarrow \mathbb{R}, u \longmapsto\left\langle\nabla h\left(-I_{a}(u)\right), u\right\rangle,
$$

where $\langle.,$.$\rangle is the standard Euclidean metric on \mathbb{R}^{4 n} \cong \mathbb{H}^{n}$, and $\nabla$ h the gradient of $h$. Thus, $\partial_{a} h$ is such that: $\forall u \in \mathbb{S}^{\prime n-1}$,

$$
\left(\partial_{a} h\right)\left(I_{a}(u)\right)=\left\langle\nabla h(u), I_{a}(u)\right\rangle=(d h)_{u}\left(I_{a}(u)\right) .
$$

The proof (very similar to that of the proposition concerning evolutes of hedgehogs in $\left.\left(\mathbb{H}, J_{v}, \omega_{v}\right),\left(v \in \mathbb{S}^{2}=\mathbb{S}^{3} \cap \operatorname{Im}(\mathbb{H})\right)\right)$ is left to the reader.

\section{Acknowledgment}

The author would like to thank the anonymous referee for carefully reading the manuscript and for providing useful and detailed comments.

\section{References}

[1] A. D. Aleksandrov, Zur Theorie der gemischten Volumina von konvexen Körpern, I:Verallgemeinerung einiger Begriffe der Theorie der konvexen Körper (in Russian). Mat. Sbornik N. S. 2 (1937), 947-972.

[2] A. Cannas da Silva, Lectures on Symplectic Geometry. Lectures Notes in Mathematics, 1764, Springer-Verlag, Berlin, 2001 and 2008.

[3] J. Duval, Around Brody, 2017: https://arxiv.org/pdf/1703.01850v1.pdf

[4] H. Geppert, Über den Brunn-Minkowskischen Satz. Math. Z. 42 (1937), $238-254$.

[5] H. Görtler, Erzeugung stützbarer Bereiche I. Deutsche Math. 2 (1937), $454-456$.

[6] H. Görtler, Erzeugung stützbarer Bereiche II. Deutsche Math. 3 (1937), 189-200.

[7] E. Grindberg and G. Zhang, Convolutions, transforms, and convex bodies. Proc. London Math. Soc. 78 (1999), 73-115.

[8] R. Langevin, G. Levitt and H. Rosenberg, Hérissons et multihérissons (enveloppes paramétrées par leur application de Gauss). In: Singularities, Banach Center Publ. 20, Warsaw, PWN, 1988, 245-253.

[9] Y. Martinez-Maure, De nouvelles inégalités géométriques pour les hérissons. Arch. Math. 72 (1999), 444-45. 
[10] Y. Martinez-Maure, Hedgehogs and zonoids. Adv. Math. 158 (2001), 1-17.

[11] Y. Martinez-Maure, Contre-exemple à une caractérisation conjecturée de la sphère. C. R. Acad. Sci. Paris, Sér. I, 332 (2001), 41-44.

[12] Y. Martinez-Maure, Geometric study of Minkowski differences of plane convex bodies. Canad. J. Math. 58 (2006), 600-624.

[13] Y. Martinez-Maure, Hedgehog theory via Euler Calculus. Beitraege zur Algebra und Geometrie 56 (2015), 397-421.

[14] D. McDuff and D. Salomon, J-holomorphic curves and symplectic topology. Colloquium Publications, American Mathematical Society 52, 2012.

[15] G. Panina, New counterexamples to A. D. Alexandrov's hypothesis. Adv. Geom. 5 (2005), 301-317.

[16] R. Schneider, Convex Bodies: The Brunn-Minkowski Theory, 2nd expanded ed. Cambridge Univ. Press, 2014.

[17] C. Wendl, Lectures on Holomorphic Curves in Symplectic and Contact Geometry, 2014: https://arxiv.org/pdf/1011.1690.pdf

Y. Martinez-Maure

Sorbonne Université et Université de Paris

Institut Mathématique de Jussieu - Paris Rive Gauche

UMR 7586 du CNRS

Bâtiment Sophie Germain

Case 7012

75205 Paris Cedex 13

France 